MATHEMATICS OF COMPUTATION

Volume 74, Number 251, Pages 1139-1177

S 0025-5718(04)01733-8

Article electronically published on December 8, 2004

\title{
BEM WITH LINEAR COMPLEXITY FOR THE CLASSICAL BOUNDARY INTEGRAL OPERATORS
}

\author{
STEFFEN BÖRM AND STEFAN A. SAUTER
}

\begin{abstract}
Alternative representations of boundary integral operators corresponding to elliptic boundary value problems are developed as a starting point for numerical approximations as, e.g., Galerkin boundary elements including numerical quadrature and panel-clustering. These representations have the advantage that the integrands of the integral operators have a reduced singular behaviour allowing one to choose the order of the numerical approximations much lower than for the classical formulations.

Low-order discretisations for the single layer integral equations as well as for the classical double layer potential and the hypersingular integral equation are considered. We will present fully discrete Galerkin boundary element methods where the storage amount and the CPU time grow only linearly with respect to the number of unknowns.
\end{abstract}

\section{INTRODUCTION}

The integral equation method is an elegant tool for transforming homogeneous linear boundary value problems with constant coefficients into boundary integral equations (BIE) on the boundary of the domain (see, e.g., [14, [19]). The boundary element method is a flexible discretisation technique for solving these equations numerically. In the last twenty years, efficient algorithms have been developed for overcoming the major bottlenecks of this method: Quadrature methods for evaluating singular and nearly singular surface integrals have been established (cf. [28], [17, [8, [26], 31]) and sparse representations for the non-local integral operators have been developed ([16], [24, 25], [31]). By employing such fast algorithms, the computational complexity and the storage amount for solving boundary integral equations were reduced from $\mathcal{O}\left(n^{2}\right)$ to $\mathcal{O}\left(n \log ^{\kappa} n\right)$, where $n$ denotes the number of degrees of freedom of the discretisation and $\kappa \sim 4-6$. Asymptotically, with increasing $n$, the term $\log ^{\kappa} n$ becomes negligible compared to $n$ while for practical problem sizes the factor $\log ^{\kappa} n$ has a significant effect on the run-time behaviour of the algorithms.

The goal of this paper is the introduction of a new, fully discrete boundary element method for all kinds of classical boundary integral equations (single layer, double layer and hypersingular operator) related to Laplace's equation,

(1) which preserves the convergence rates of the "true" Galerkin BEM with respect to the energy norm while

Received by the editor September 9, 2003 and, in revised form, March 29, 2004.

2000 Mathematics Subject Classification. Primary 65N38, 65D05.

Key words and phrases. BEM, data-sparse approximation, $\mathcal{H}^{2}$-matrices.

This work was supported by the Swiss National Science Foundation, Grant 21-6176400. 
(2) the computational complexity and storage amount is $\mathcal{O}(n)$ without any logarithmic factors.

The method is based on the combination of the following ideas:

- Alternative representations of classical boundary integral operators are derived which reduce the singular behaviour of the kernel function.

- The reduced singular behaviour of the kernel function allows the approximation of the diagonal and near-diagonal entries of the system matrix by low-order quadrature methods. In some cases, even the replacement by 0 is stable and consistent! No complicated evaluations of singular surface integrals are necessary any more (cf. [3]).

- A modified version of the panel-clustering method with variable approximation order will be introduced which allows the representation of the farfield part of the system matrix in a sparse way. The changes from the classical panel-clustering algorithm to the new one are only moderate. Various shift and summation operations simply have to be cut earlier than in the classical versions.

We will prove that this fully discrete Galerkin BEM satisfies the goals 1 and 2 formulated above. The main results are the alternative representations (3.3), (3.8), and (3.10) of the integral operators, the panel-clustering approximations of the arising bilinear forms (4.34), the choice of the expansion orders (4.33), (6.14), the algorithmic description of the method at the end of Section 5 and the justification of the choices of the expansion orders by the error analysis in Section 6 . The proof that the complexity of the method is $\mathcal{O}(n)$ is in Section 7 .

We emphasize that all existing multipole and panel-clustering-type methods applied, e.g., to the classical formulation of the single layer operator on, possibly, curved and only piecewise smooth surfaces, in general, cannot perform better than $\mathcal{O}\left(n \log ^{4} n\right)$ if the asymptotic convergence rate with respect to the energy norm of the unperturbed Galerkin method has to be preserved. (Among other reasons, this follows theoretically from the error analysis of the quadrature method for the nearly singular integrals in Section 6.5.2 and the sharpness of these estimates is demonstrated by numerical experiments in [3].) Hence, the asymptotic gain in complexity of the new panel-clustering method compared to other methods is obvious.

It is worth noting that the existing multipole and panel-clustering methods sometimes are applied as $O(n)$-algorithms by fixing the quadrature and expansion order independently of $n$. However, e.g., for the single layer operator this leads to a reduced convergence rate with respect to the energy norm.

The important question at what problem sizes the new method performs more efficiently than, e.g., multipole methods strongly depends on the constants that are hidden in the $\mathcal{O}(\cdot)$-estimates and the implementation. The implementation of the new panel-clustering method is the topic of current research, and we will publish the results of numerical experiments in a forthcoming paper.

In our paper, the approximation of the kernel function is based on Chebyshev interpolation of some generator functions. Further research directions will address the approximation of these generator functions in our alternative representations by multipole-type methods. Thus, we expect that the constants in the complexity estimates can be reduced further. 
The paper is at some point a bit technical since the detailed analysis and subtle combination of all these ingredients are essential to achieve the prescribed goal.

\section{Galerkin discretisation of Integral operators}

Throughout this paper, $\Omega \subset \mathbb{R}^{3}$ denotes a bounded Lipschitz domain with boundary $\Gamma$ and normal vector field $n$ (oriented to the exterior of $\Omega$ ). We define the Sobolev space $H^{s}(\Gamma), s \geq 0$, in the usual way (see, e.g., [19]). Note that the range of $s$ for which $H^{s}(\Gamma)$ is defined may be limited, depending on the global smoothness of the surface $\Gamma$. For $s<0$, the spaces $H^{s}(\Gamma)$ are the dual of $H^{-s}(\Gamma)$.

We will consider the general integral equation

$$
(\lambda I+\mathcal{K}) u(x):=\lambda u(x)+\int_{\Gamma} k(x, y) u(y) d s_{y}=f(x), \quad x \in \Gamma,
$$

for some given scalar $\lambda \in \mathbb{R}$, kernel function $k$ and sufficiently smooth right-hand side $f$. The corresponding weak form is

$$
\text { Find } u \in H \text { such that } a(u, v):=((\lambda I+\mathcal{K}) u, v)=(f, v) \text { for all } v \in H \text {. }
$$

Here $H$ equals $H^{\mu}(\Gamma)$ or is a closed subspace of $H^{\mu}(\Gamma)$ for some $\mu \in\{-1 / 2,0,1 / 2\}$. (The bracket $(\cdot, \cdot)$ denotes the continuous extension of the $L^{2}(\Gamma)$ scalar product to the $H^{-\mu}(\Gamma) \times H^{\mu}(\Gamma)$ duality pairing.) Typical examples are: the classical single layer, double layer and hypersingular operators for the Laplacian where $a(\cdot, \cdot)=$ $\lambda(\cdot, \cdot)+\hat{a}(\cdot, \cdot)$ with:

\section{Single layer potential:}

$$
\lambda=0, H=H^{-1 / 2}(\Gamma), \hat{a}(u, v):=\int_{\Gamma \times \Gamma} \frac{v(x) u(y)}{4 \pi\|x-y\|} d s_{x} d s_{y},
$$

\section{Double layer potential:}

$$
\lambda= \pm \frac{1}{2}, H=L^{2}(\Gamma), \hat{a}(u, v):=\int_{\Gamma \times \Gamma} v(x) u(y) \frac{\partial}{\partial n_{y}} \frac{1}{4 \pi\|x-y\|} d s_{y} d s_{x},
$$

\section{Hypersingular operator:}

$$
\lambda=0, H=H^{1 / 2}(\Gamma) / \mathbb{R}, \hat{a}(u, v):=\int_{\Gamma} v(x) \frac{\partial}{\partial n_{x}} \int_{\Gamma} u(y) \frac{\partial}{\partial n_{y}} \frac{1}{4 \pi\|x-y\|} d s_{y} d s_{x} .
$$

In the standard, conforming Galerkin method we select a subspace $\mathcal{S} \subset H$ and approximate (2.2) by seeking $U \in \mathcal{S}$, such that

$$
a(U, V)=(f, V) \quad \text { for all } V \in \mathcal{S} .
$$

In the context of the boundary element method, these subspaces are finite element spaces lifted to the surface $\Gamma$.

Definition 2.1. $\quad$ (a) The master element $\hat{t} \subseteq \mathbb{R}^{2}$ is the open triangle with vertices $(0,0)^{\top},(0,1)^{\top}$ and $(1,1)^{\top}$.

(b) A set $\mathcal{T}=\left\{t_{1}, t_{2}, \ldots, t_{n}\right\}$ consisting of open and disjoint (possibly curved) triangles in $\mathbb{R}^{3}$ such that there is a $C^{k}$-diffeomorphism $\Psi_{t}: \overline{\hat{t}} \rightarrow \bar{t}$ for each $t \in \mathcal{T}$ is a surface triangulation of $\Gamma$ if it satisfies

$$
\Gamma=\bigcup_{t \in \mathcal{T}} \bar{t}
$$


(c) The triangulation is compatible if the intersection $\bar{t} \cap \overline{t^{\prime}}=: e$ of non-identical triangles $t, t^{\prime} \in \mathcal{T}$ is either empty, a common vertex, or a common edge and, in the case that $e$ is an edge, there exists an affine mapping $\gamma: \overline{\hat{t}} \rightarrow \overline{\hat{t}}$ such that $\left.\Psi_{t}^{-1}\right|_{e}=\left.\left(\Psi_{t^{\prime}} \circ \gamma\right)^{-1}\right|_{e}$.

The step size of a grid is given by

$$
h=\max _{t \in \mathcal{T}} \operatorname{diam}(t) .
$$

For $k \in\{0,1\}$ and $p \in \mathbb{N}_{0}$, the finite element space $\mathcal{S}(k, p, \mathcal{T})$ is defined by

$$
\mathcal{S}(k, p, \mathcal{T}):=\left\{u \in H^{k}(\Gamma) \mid \forall t \in \mathcal{T}: u \circ \Psi_{t} \in \mathbb{P}_{p}\right\}
$$

Remark 2.2. If $k=1$, we assume throughout the paper that the triangulation is compatible.

We will consider boundary elements of lowest order for the discretisation of the integral operators, i.e.,

- $\mathcal{S}=\mathcal{S}(0,0, \mathcal{T})$ for the single layer operator and

- $\mathcal{S}=\mathcal{S}(1,1, \mathcal{T}) / \mathbb{R}$ for the hypersingular operator.

- The boundary element space for the double layer potential operator is a subset of $\mathcal{S}(0,0, \mathcal{T})$ that contains all functions vanishing in a certain neighbourhood of the corners and edges of the surface $\Gamma$. For the detailed definition of the finite section method we refer to [7].

Let $\left(b_{i}\right)_{i=1}^{n}$ be the local basis of $\mathcal{S}(k, p, \mathcal{T})$. Hence, every function $u \in \mathcal{S}$ is characterised uniquely by the coefficient vector $\mathbf{u}=\left(u_{i}\right)_{i=1}^{n} \in \mathbb{R}^{n}$ with respect to this basis:

$$
u=\sum_{i=1}^{n} u_{i} b_{i}
$$

If $u \in \mathcal{S}$ and $\mathbf{u} \in \mathbb{R}^{n}$ appear in the same context we will assume throughout the paper that (2.6) holds. Plugging this representation into equation (2.4) and testing by the basis functions, we can rewrite the variational equation as a system of linear equations

$$
\mathbf{K u}=\mathbf{F}
$$

with

$$
\mathbf{K}_{i j}=a\left(b_{i}, b_{j}\right) \quad \text { and } \quad \mathbf{F}=F\left(b_{j}\right)
$$

for $i, j \in\{1, \ldots, n\}$.

The basis representation of the Galerkin method transforms the continuous problem into a system of linear equations. The direct numerical realisation of this approach suffers from two bottlenecks: (a) the computation of the matrix entries requires the evaluation of singular, nearly singular and regular surface integrals over pairs of panels; (b) the system matrix is not sparse but fully populated and the computational and storage costs are at least of order $n^{2}$. 


\section{Alternative representation \\ OF CLASSICAL BOUNDARY INTEGRAL OPERATORS}

In this section, we will derive an alternative representation of classical boundary integral equations, where the kernels are replaced by tangential derivatives of some generator functions which have reduced singular behaviour. In our applications this generator function is one of

$$
G_{1}(x, y):=\frac{1}{4 \pi\|x-y\|}, \quad G_{2}(x, y):=\frac{\|x-y\|}{4 \pi} .
$$

(Note that $G_{1}$ is the fundamental solution of the Laplace operator in three dimensions.)

The advantage compared to the classical formulations, which was based on a polynomial (globally discontinuous) expansion of the kernel functions, is as follows. If we replace the generator function $G$ on $\Gamma \times \Gamma$ by some globally Lipschitz continuous approximation $\tilde{G} \in C^{0,1}(\Gamma \times \Gamma)$, we may obtain an approximation $\tilde{k}$ of the kernel function $k$ by applying the tangential derivative, say $D$, to $\tilde{G}$. Typically, the approximation $G-\tilde{G}$ is of higher order compared to the approximation of $k-\tilde{k}=D(G-\tilde{G})$. For the error analysis, we apply partial integration, pulling the derivative $D$ to the test and trial function and may take advantage of the possible regularity of the exact solution. The continuity of $G-\tilde{G}$ avoids the appearance of line integrals due to partial integration. Thus, in the error estimates the difference $G-\tilde{G}$ instead of $k-\tilde{k}$ enters allowing us to reduce the order of approximation.

3.1. Classical double layer potential. It is well known that

$$
\Upsilon_{\Gamma}: \Gamma \rightarrow \mathbb{R}, \quad x \mapsto-\int_{\Gamma} \frac{\partial}{\partial n_{y}} G_{1}(x, y) d s_{y}=-\frac{1}{4 \pi} \int_{\Gamma} \frac{\left\langle n_{y}, x-y\right\rangle}{\|x-y\|^{3}} d s_{y}
$$

defines a function in $L^{2}(\Gamma)$ that has constant value $1 / 2$ almost everywhere on $\Gamma$. Hence, the constant function $1 / 2$ on $\Gamma$ coincides in the $L^{2}$-sense with $\Upsilon_{\Gamma}$.

This means that we can rewrite the bilinear form $a_{D}$ of the double layer potential (cf. (2.3b)) as

$$
a_{D}(u, v)=\left(\lambda-\frac{1}{2}\right)(u, v)+\int_{\Gamma \times \Gamma} v(x)(u(y)-u(x)) \frac{\partial G_{1}(x, y)}{\partial n_{y}} d s_{y} d s_{x}
$$

for all $u, v \in L^{2}(\Gamma)$. The difference to the standard form in $2.3 \mathrm{~b}$ ) is that the integrand of the second term in (3.3) has a reduced singular behaviour if the function $u$ has some regularity, e.g., $u \in H^{1}(\Gamma)$.

3.2. Hypersingular operator. Partial integration in the form of Stokes' theorem can be applied to equation (2.3c) for Lipschitz surfaces (cf. 21], 222) to obtain, for the bilinear form $a_{H}$ of the hypersingular operator,

$$
a_{H}(u, v)=\int_{\Gamma \times \Gamma}\left\langle\overrightarrow{\operatorname{curl}}_{\Gamma} v(x), \overrightarrow{\operatorname{curl}}_{\Gamma} u(y)\right\rangle G_{1}(x, y) d s_{y} d s_{x} .
$$

Here, the surface curl $\overrightarrow{\operatorname{curl}}_{\Gamma}$ is a tangential differential operator. For functions $u \in$ $H^{1 / 2}(\Gamma)$ having an extension $u^{\star} \in H^{1}(\mathcal{U})$ into a three-dimensional neighbourhood $\mathcal{U}$ of $\Gamma$, the surface gradient $\nabla_{\Gamma}$ and the surface curl are defined by

$$
\nabla_{\Gamma} u:=\left.\left(\nabla u^{\star}\right)\right|_{\Gamma} \quad \text { and } \quad \overrightarrow{\operatorname{curl}}_{\Gamma} u:=-n \times \nabla_{\Gamma} u \quad \text { on } \Gamma .
$$


For a differentiable vector field $F: \mathbb{R}^{3} \rightarrow \mathbb{R}^{3}$, we introduce the scalar counterpart of the surface curl by

$$
\operatorname{curl}_{\Gamma} F:=\langle n, \operatorname{curl} F\rangle \quad \text { on } \Gamma,
$$

where curl denotes the curl operator in the Euclidean space $\mathbb{R}^{3}$. The composition of the surface curl and tangential curl operators leads to the Laplace-Beltrami operator

$$
\Delta_{\Gamma} u=-\operatorname{curl}_{\Gamma} \overrightarrow{\operatorname{curl}}_{\Gamma} u=\left.\left(\Delta u^{*}-\frac{\partial^{2}}{\partial n^{2}} u^{*}\right)\right|_{\Gamma} .
$$

Simple tensor analysis yields

$$
\frac{1}{\|y-x\|}=\Delta_{\Gamma, y}\|y-x\|+\left\langle n_{y}, y-x\right\rangle \frac{\partial}{\partial n_{y}} \frac{1}{\|y-x\|}
$$

for all $y \in \Gamma$ and $x \in \mathbb{R}^{3} \backslash\{y\}$. The second derivatives in $\Delta_{\Gamma, y}$ with respect to $y$ would complicate the panel-clustering method and require higher global smoothness of the approximation. Thus, we express the Laplace-Beltrami operator (3.6) by a composition of first-order surface derivatives with respect to $x$ and $y$.

Lemma 3.1. Let $\nabla_{\Gamma}^{t}:=\nabla_{\Gamma}-n \partial / \partial n$ denote the tangential gradient and let $\Delta_{\Gamma, x y}:=$ $-\left\langle\nabla_{\Gamma, x}, \nabla_{\Gamma, y}^{t}\right\rangle$. Then, for $G_{2}$ as in (3.1), it follows that

$$
\Delta_{\Gamma, y} G_{2}(x, y)=\Delta_{\Gamma, x y} G_{2}(x, y) \quad \forall y \in \Gamma, \forall x \in \mathbb{R}^{3} \backslash\{y\} .
$$

Proof. The proof follows from

$$
\begin{aligned}
\Delta_{\Gamma, y}\|x-y\| & =\left.\left(\operatorname{curl}_{\Gamma, y}\left(n_{z} \times \nabla_{y}\|x-y\|\right)\right)\right|_{z=y}=-\left.\left(\operatorname{curl}_{\Gamma, y}\left(n_{z} \times \nabla_{x}\|x-y\|\right)\right)\right|_{z=y} \\
& =\left\langle\nabla_{x}, n_{y} \frac{\partial}{\partial n_{y}}-\nabla_{y}\right\rangle\|x-y\|=-\left\langle\nabla_{x}, \nabla_{\Gamma, y}^{t}\right\rangle\|x-y\| .
\end{aligned}
$$

Remark 3.2. For a differentiable function $F: U_{\Gamma} \rightarrow \mathbb{R}$ that is defined in a tubular neighbourhood $U_{\Gamma}$ of $\Gamma$ we introduce (cf. (3.5)) the mixed surface curl by

$$
\overrightarrow{\operatorname{curl}}_{\Gamma, x y}:=-n_{y} \times \nabla_{\Gamma, x}
$$

and obtain

$$
\Delta_{\Gamma, x y}=\operatorname{curl}_{\Gamma, y} \overrightarrow{\operatorname{curl}}_{\Gamma, x y} .
$$

Replacing the kernel in (3.4) by (B.6), we obtain the representation

$$
\begin{aligned}
& a_{H}(u, v) \\
& =\int_{\Gamma} \int_{\Gamma}\left\langle\overrightarrow{\operatorname{curl}}_{\Gamma} u(y), \overrightarrow{\operatorname{curl}}_{\Gamma} v(x)\right\rangle\left(\Delta_{\Gamma, x y} G_{2}(x, y)+\left\langle n_{y}, y-x\right\rangle \frac{\partial G_{1}}{\partial n_{y}}(x, y)\right) d s_{y} d s_{x} .
\end{aligned}
$$


3.3. Single layer potential. In order to work out the principal idea for the regularization of the single layer potential (2.3a), we assume that the Lipschitz boundary $\Gamma$ is the surface of a polyhedron, i.e., there exist disjoint open plane faces $\Gamma_{i}$, $i \in\{1, \ldots, q\}$, such that

$$
\Gamma=\bigcup_{i=1}^{q} \overline{\Gamma_{i}} .
$$

The oriented distance of $x$ from the extended infinite plane $\Gamma_{i}^{\star}$ through $\Gamma_{i}$ is given by

$$
d_{i}(x):=\left\langle n_{i}, x-c_{i}\right\rangle
$$

where $n_{i}$ is the constant outer normal vector on $\Gamma_{i}$ and $c_{i} \in \Gamma_{i}$. A simple consequence is that

$$
\left\langle n_{y}, x-y\right\rangle=d_{i}(x)
$$

holds for all $y \in \Gamma_{i}$.

Due to (3.6), the bilinear form $a_{S}$ for the single layer operator (2.3a) has the representation

$$
\begin{aligned}
a_{S}(u, v)= & \int_{\Gamma} \int_{\Gamma} v(x) G_{1}(x, y) u(y) d s_{y} d s_{x} \\
= & \frac{1}{4 \pi} \int_{\Gamma} \int_{\Gamma} v(x) u(y)\left(\Delta_{\Gamma, x y}\|y-x\|+\left\langle n_{y}, y-x\right\rangle \frac{\partial}{\partial n_{y}} \frac{1}{\|y-x\|}\right) d s_{y} d s_{x} \\
= & \int_{\Gamma} \int_{\Gamma} v(x) u(y) \Delta_{\Gamma, x y} G_{2}(x, y) d s_{y} d s_{x} \\
& +\int_{\Gamma} \int_{\Gamma} v(x)(u(y)-u(x))\left\langle n_{y}, y-x\right\rangle \frac{\partial}{\partial n_{y}} G_{1}(x, y) d s_{y} d s_{x} \\
& +\int_{\Gamma} v(x) u(x) \sum_{i=1}^{q} \int_{\Gamma_{i}}\left\langle n_{y}, y-x\right\rangle \frac{\partial}{\partial n_{y}} G_{1}(x, y) d s_{y} d s_{x} .
\end{aligned}
$$

By using (3.9), we can simplify the last term in order to get

$$
\begin{aligned}
a_{S}(u, v)= & \int_{\Gamma} \int_{\Gamma} v(x) u(y) \Delta_{\Gamma, x y} G_{2}(x, y) d s_{y} d s_{x} \\
& +\int_{\Gamma} \int_{\Gamma} v(x)(u(y)-u(x))\left\langle n_{y}, y-x\right\rangle \frac{\partial}{\partial n_{y}} G_{1}(x, y) d s_{y} d s_{x} \\
& +(\rho u, v),
\end{aligned}
$$

where $\rho(x):=\sum_{i=1}^{q} d_{i}(x) \Upsilon_{i}(x)$ is a weight function and the spherical angle $\Upsilon_{i}(x)$ is given by

$$
\Upsilon_{i}(x):=-\int_{\Gamma_{i}} \frac{\partial}{\partial n_{y}} G_{1}(x, y) d s_{y} .
$$

Note that, for polyhedral surfaces, $\Upsilon_{i}$ can be computed analytically (see, e.g., 9]).

\section{KeRnel APPROXIMATION}

A matrix-vector multiplication appears as a basic arithmetic operation in every step of an iterative solution method for solving the linear system in (2.7). The result of a multiplication of the system matrix corresponding to a bilinear form $a(\cdot, \cdot)$ with a vector $\mathbf{u}$ is $\left(a\left(b_{i}, u\right)\right)_{i=1}^{n}$, where $b_{i}$ denotes the basis of the boundary element space and $u$ is the boundary element function corresponding to the coefficient vector $\mathbf{u}$. 
The representation of the Galerkin discretisation with respect to the nodal basis of the boundary element space leads to a full matrix and the computational and storage costs of assembling the matrix and of a matrix-vector multiplication are of order $n^{2}$. The panel-clustering method allows us to represent the Galerkin discretisation with $O\left(n \log ^{\kappa} n\right)$ quantities in a non-matrix form (our new approach results in $\kappa=0$ ). This representation allows the efficient evaluation of a matrix-vector multiplication and, hence, iterative solvers can be employed for solving the linear systems.

All kernels in the bilinear forms $a_{S}, a_{D}, a_{H}$ (cf. (3.10), (3.3), (3.8)) are Gâteaux derivatives of scalar generator functions defined in $\mathbb{R}^{3}$. Our concept for the kernel approximation is to approximate the generator functions and then to approximate the kernel by the Gâteaux derivative of the expansion.

4.1. Generalized cluster tree. The standard panel-clustering method is based on the local approximation of the kernel function by a degenerate kernel on nonoverlapping (six-dimensional) subsets of the domain $\Gamma \times \Gamma$. Since we need a globally continuous approximation (cf. Section [3), we would have to ensure continuity along the one- to five-dimensional intersections of the respective subsets, which would lead to a complicated algorithm.

Instead, we construct approximations on overlapping subsets of $\Gamma \times \Gamma$ and blend them by using a partition of unity $\left(w^{(\sigma, s)} \chi^{(\sigma, s)}\right)_{(\sigma, s) \in P}$ consisting of non-negative, globally continuous cutoff functions $\chi^{(\sigma, s)} \in C^{0,1}(\Gamma \times \Gamma)$ and positive weights $w^{(\sigma, s)} \subset \mathbb{R}$.

Thus, any function $f: \Gamma \times \Gamma \rightarrow \mathbb{R}$ has the representation

$$
f(x, y)=\sum_{(\sigma, s) \in P} w^{(\sigma, s)} \chi^{(\sigma, s)}(x, y) f(x, y) .
$$

The construction of the functions $\chi^{(\sigma, s)}$ will result in a tensor structure, i.e., $\chi^{(\sigma, s)}=\chi^{\sigma} \otimes \chi^{s}$ with functions $\chi^{\sigma}, \chi^{s} \in C^{0,1}(\Gamma)$ that have local support. In our application, the function $f$ will be the generator function $G \in\left\{G_{1}, G_{2}\right\}$. If, for $\mathbf{b}=(\sigma, s) \in P$, the supports of $\chi^{\sigma}$ and $\chi^{s}$ are well separated, the kernel function can be approximated by a degenerate expansion.

Definition 4.1 (Hierarchical partition of unity). A function system

$$
\left(\chi^{\sigma}\right)_{\sigma \in T} \quad \text { with } \quad \chi^{\sigma}: \Gamma \rightarrow \mathbb{R} \text { for all } \sigma \in T
$$

along with a disjoint partition $T=\left(T_{\ell}\right)_{\ell=0}^{L}$ of the index set $T$ in (4.1) is a hierarchical partition of unity if

(1) for all $\ell \in\{0, \ldots, L\}$, the family $\left(\chi^{\sigma}\right)_{\sigma \in T_{\ell}}$ is a partition of unity for $\Gamma$,

(2) for each $\ell \in\{0, \ldots, L-1\}$ and each $\sigma \in T_{\ell}$, there are a set sons $(\sigma) \subset T_{\ell+1}$ and positive coefficients $\left(\gamma^{\sigma, \sigma^{\prime}}\right)_{\sigma^{\prime} \in \operatorname{sons}(\sigma)}$ satisfying

$$
\chi^{\sigma}=\sum_{\sigma^{\prime} \in \operatorname{sons}(\sigma)} \gamma^{\sigma, \sigma^{\prime}} \chi^{\sigma^{\prime}} .
$$

The level of a cluster $\sigma \in T_{\ell}$ is denoted by level $(\sigma):=\ell$. The elements in $T$ are called clusters. In contrast to the classical panel-clustering method the set $T$ is not a tree but defines a simple hierarchy of clusters via the relation $\sigma \rightarrow \operatorname{sons}(\sigma)$. 

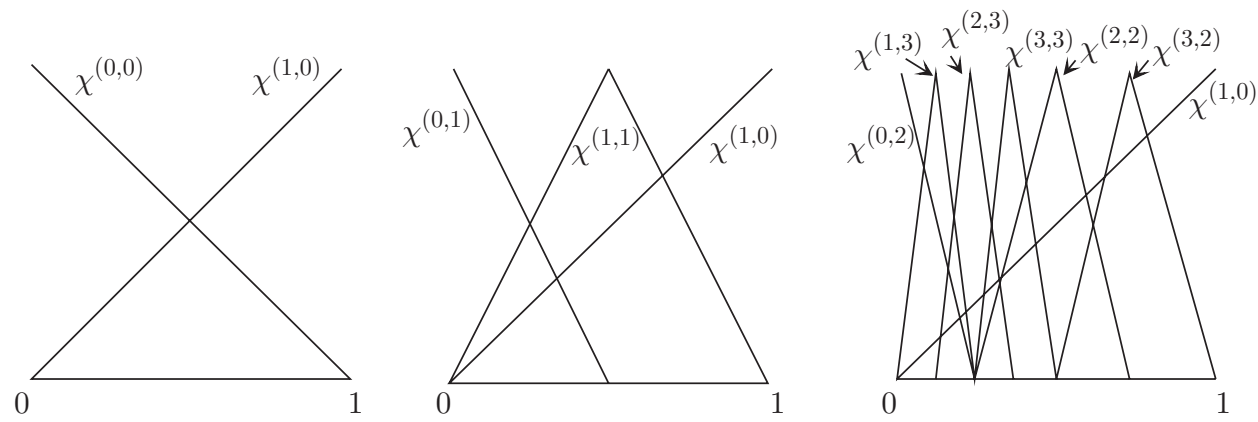

Figure 1. Cutoff functions arising in the multiscale representations 1, 2, 4 of $f$ as in (4.3). The sizes and overlaps of their supports are related to the different refinement levels of the meshes where they are defined.

Remark 4.2. For the classical panel-clustering method, the clusters are subsets of the set of freedoms for the boundary element discretisation and the hierarchy of clusters is defined via set inclusion. The equation (4.2) is the analogon of this condition.

Example 4.3. Let $\Gamma=(0,1)$ and, for $\ell=0,1, \ldots, L$, define the index sets $T_{\ell}:=$ $\left\{(\mu, \ell): 0 \leq \mu \leq n_{\ell}\right\}$ where $n_{\ell}=2^{\ell}$.

For $0 \leq \ell \leq L$, let $\left(x_{\mu, \ell}\right)_{\mu=0}^{n_{\ell}}:=\left(\mu / n_{\ell}\right)_{\mu=0}^{n_{\ell}}$ denote the set of equidistant grid points and let $\left(\chi^{\sigma}\right)_{\sigma \in T_{\ell}}$ be the continuous, piecewise linear Lagrange basis corresponding to the nodal points $\left(x_{\sigma}\right)_{\sigma \in T_{\ell}}$.

The function system $\left(\chi^{\sigma}\right)_{\sigma \in T_{\ell}}$ forms a $C^{0,1}(\Gamma)$-partition of unity for $\Gamma$ and satisfies, for $\sigma=(\mu, \ell) \in T_{\ell}$ with $0 \leq \ell<L$, the relation

$$
\chi^{\sigma}=\sum_{\sigma^{\prime} \in \operatorname{sons}(\sigma)} \gamma^{\sigma, \sigma^{\prime}} \chi^{\sigma^{\prime}}
$$

with sons $(\sigma)=\{(2 \mu-1, \ell+1),(2 \mu, \ell+1),(2 \mu+1, \ell+1)\} \cap T_{\ell+1}$ and

$$
\gamma^{\sigma, \sigma^{\prime}}:=\left\{\begin{array}{ll}
1 & x_{\sigma}=x_{\sigma^{\prime}} \\
1 / 2 & \text { otherwise }
\end{array}\right\} \quad \forall \sigma^{\prime} \in \operatorname{sons}(\sigma) .
$$

There exist numerous (multiscale) decompositions of a function $f: \Gamma \rightarrow \mathbb{R}$ with respect to a prescribed subset $P \subset T$; some examples are shown below and illustrated in Figure 1:

$$
\begin{aligned}
f & =\chi^{(0,0)} f+\chi^{(1,0)} f=\chi^{(0,1)} f+\frac{1}{2} \chi^{(1,1)} f+\chi^{(1,0)} f \\
& =\chi^{(0,2)} f+\frac{3}{4} \chi^{(1,2)} f+\frac{1}{2} \chi^{(2,2)} f+\frac{1}{4} \chi^{(3,2)} f+\chi^{(1,0)} f \\
& =\chi^{(0,2)} f+\frac{3}{8} \chi^{(1,3)} f+\frac{3}{4} \chi^{(2,3)} f+\frac{3}{8} \chi^{(3,3)} f+\frac{1}{2} \chi^{(2,2)} f+\frac{1}{4} \chi^{(3,2)} f+\chi^{(1,0)} f .
\end{aligned}
$$

Remark 4.4. The generalisation of the approach of Example4.3 for the construction of hierarchical partitions of unity to sequences $\left(\mathcal{G}_{\ell}\right)_{\ell=0}^{L}$ of nested surface triangulations is straightforward.

Note that the definition of a hierarchy of partitions of unity does not necessarily require a sequence of nested meshes. One might employ techniques developed in 
the fields of composite finite elements (cf. [18]), agglomeration methods (cf. [1], [30]), the partition of unity method (PUM) (cf. [20]) or meshless methods (cf. [13]) for this purpose.

For the efficiency of the algorithm, it is essential that there exists a constant $C_{\text {sons }} \in \mathbb{N}$ such that

$$
|\operatorname{sons}(\sigma)| \leq C_{\text {sons }}
$$

holds for all $\sigma \in T$.

We will use tensor-product interpolation on axis-parallel boxes $Q^{\sigma} \subset \mathbb{R}^{3}$ satisfying the condition

$$
\operatorname{supp} \chi^{\sigma} \subseteq Q^{\sigma}
$$

for the construction of the kernel approximation. In order to find a stable interpolation scheme, we require that the partition of unity consists of functions in $W^{1, \infty}(\Gamma)$ and that there are constants $C_{\text {stab }}, C_{\text {supp }}, C_{\sharp} \in \mathbb{R}_{>0}$ such that

$$
\begin{aligned}
& \left\|\chi^{\sigma}\right\|_{W^{1, \infty}(\Gamma) \leq C_{\mathrm{stab}} 2^{\operatorname{level}(\sigma)},} \quad \operatorname{diam} Q^{\sigma} \leq C_{\mathrm{supp}} 2^{-\operatorname{level}(\sigma)}, \\
& \max _{\tau \in \mathcal{T}} \operatorname{card}\left\{\sigma \in T_{L}: \tau \subset \operatorname{supp} \chi^{\sigma}\right\} \leq C_{\sharp},
\end{aligned}
$$

where card $\{\ldots\}$ denotes the cardinality of a set.

Furthermore, we assume that there is an extension $\chi_{+}^{\sigma}$ of $\chi^{\sigma}$ into a tubular neighbourhood of $\Gamma$ such that the normal derivative $\partial \chi_{+}^{\sigma} / \partial n$ is in $L^{\infty}(\Gamma)$ and

$$
\partial \chi_{+}^{\sigma} / \partial n=0 \quad \text { almost everywhere on } \Gamma \text {. }
$$

In the following, we skip the index "+" in $\chi_{+}^{\sigma}$ and identify $\chi^{\sigma}$ with its extension.

4.2. Generalized partitions. The kernel function in (2.1) that we want to approximate is defined on $\Gamma \times \Gamma$, while $T$ corresponds to partitions of unity for $\Gamma$. We will use a tensor-product approach in order to construct a suitable partition of unity for $\Gamma \times \Gamma$ : We set $T_{\ell}^{2}:=T_{\ell} \times T_{\ell}$ and $T^{2}:=\bigcup_{\ell=0}^{L} T_{\ell}^{2}$. The elements in $T^{2}$ are called blocks. Note that, for each $\mathbf{b}=(\sigma, s) \in T^{2}$, the clusters $\sigma, s$ belong to the same level and we define level $(\mathbf{b}):=\operatorname{level}(\sigma)=\operatorname{level}(s)$. For all $\mathbf{b}=(\sigma, s) \in T^{2}$, let

$$
\chi^{\mathbf{b}}:=\chi^{\sigma} \otimes \chi^{s} \quad \text { and } \quad \operatorname{sons}(\mathbf{b}):= \begin{cases}\operatorname{sons}(\sigma) \times \operatorname{sons}(s) & \text { if level }(\mathbf{b})<L, \\ \emptyset & \text { if level }(\mathbf{b})=L .\end{cases}
$$

In order to approximate the kernel function, we need a covering of the domain $\Gamma \times \Gamma$. It will turn out in the analysis of the interpolation error that the relative distance of a pair of boxes has to be controlled by some parameter.

Definition 4.5 (Admissibility). A pair $(\sigma, s) \in T \times T$ is called $\eta$-admissible for $\eta \in \mathbb{R}_{>0}$ if

$$
\operatorname{diam}\left(Q^{\sigma} \times Q^{s}\right) \leq \eta \operatorname{dist}\left(Q^{\sigma}, Q^{s}\right) .
$$

In order to derive an efficient approximation of the integral kernels, we will specify a minimal index set $P \subset T^{2}$ along with corresponding weights $\left(w^{\mathbf{b}}\right)_{\mathbf{b} \in P}$ in $\mathbb{R}$ such that $\left(w^{\mathbf{b}} \chi^{\mathbf{b}}\right)_{\mathbf{b} \in P}$ is a partition of unity for $\Gamma \times \Gamma$ and each $\mathbf{b} \in P$ is either $\eta$-admissible or a leaf with level $(\mathbf{b})=L$. 
Definition 4.6 (Admissible partition of unity). A family $\left(w^{\mathbf{b}}\right)_{\mathbf{b} \in P} \subset \mathbb{R}_{\geq 0}$ along with an index set $P \subset T^{2}$ is an admissible weight corresponding to $\eta \in \mathbb{R}_{>0}$, if

$$
\sum_{\mathbf{b} \in P} w^{\mathbf{b}} \chi^{\mathbf{b}} \equiv 1
$$

holds and if each block $\mathbf{b}=(\sigma, s) \in P$ with level $(\mathbf{b})<L$ is $\eta$-admissible. If $\left(w^{\mathbf{b}}\right)_{\mathbf{b} \in P}$ is an admissible weight, then $\left(w^{\mathbf{b}} \chi^{\mathbf{b}}\right)_{\mathbf{b} \in P}$ is an admissible partition of unity for $\Gamma \times \Gamma$.

The weights are the tensor versions of the weighting factors that appear, e.g., in the decompositions (4.3). The procedure subdivide computes a minimal admissible weight. It is called by

$w:=0 ; P_{\text {far }}:=\emptyset ; P_{\text {near }}:=\emptyset ;$ for $\mathbf{b} \in T_{0} \times T_{0}$ do $\operatorname{subdivide~}\left(\mathbf{b}, P_{\text {near }}, P_{\text {far }}, 1, w\right) ;$

and defined by

$$
\begin{aligned}
& \text { procedure subdivide }\left(\mathbf{b}, P_{\text {near }}, P_{\text {far }}, c, w\right) \\
& \text { begin } \\
& \text { if } \mathbf{b} \text { is admissible then begin } \\
& \quad w^{\mathbf{b}}:=w^{\mathbf{b}}+c ; \quad P_{\text {far }}:=P_{\text {far }} \cup\{\mathbf{b}\} \\
& \text { end else if level }(\mathbf{b})=L \text { then begin } \\
& \quad w^{\mathbf{b}}:=w^{\mathbf{b}}+c ; \quad P_{\text {near }}:=P_{\text {near }} \cup\{\mathbf{b}\} ; \\
& \text { end else for } \mathbf{b}^{\prime} \in \operatorname{sons}(\mathbf{b}) \text { do subdivide }\left(\mathbf{b}^{\prime}, P_{\text {near }}, P_{\text {far }}, \gamma^{\sigma, \sigma^{\prime}} \gamma^{s, s^{\prime}} c, w\right) \text {; } \\
& \text { end; }
\end{aligned}
$$

Here, we used the conventions $\mathbf{b}=(\sigma, s), \mathbf{b}^{\prime}=\left(\sigma^{\prime}, s^{\prime}\right)$ and the definition of the coefficients $\gamma^{\sigma, \sigma^{\prime}}$ as in (4.2).

The index set $P \subset T^{2}$ is the union of $P_{\text {far }}$ and $P_{\text {near }}$. Note that all blocks in $P_{\text {far }}$ are $\eta$-admissible and all blocks in $P_{\text {near }}$ belong to the finest level: level $(\mathbf{b})=L$. The supports supp $\chi^{\mathbf{b}} \subset \Gamma \times \Gamma$ corresponding to $\mathbf{b}=(\sigma, s) \in P$ form a covering of $\Gamma \times \Gamma$ since $\sum_{\mathbf{b} \in P} w^{\mathbf{b}} \chi^{\mathbf{b}} \equiv 1$.

4.3. Construction of the kernel approximation. Let us first consider the standard polynomial approximation of the kernel. Let $\mathbb{D}:=\left\{(x, y) \in \mathbb{R}^{3} \times \mathbb{R}^{3}: x \neq y\right\}$. The idea is to approximate the generator functions (cf. (3.1)) $G_{1}: \mathbb{D} \rightarrow \mathbb{R}$, $G_{2}: \mathbb{D} \rightarrow \mathbb{R}$

$$
G_{1}(x, y)=\frac{1}{4 \pi\|x-y\|} \quad \text { and } \quad G_{2}(x, y)=\frac{\|x-y\|}{4 \pi}
$$

on domains $Q_{\sigma} \times Q_{s}$ corresponding to admissible blocks $(\sigma, s) \in P_{\text {far }}$.

Let $\left(x_{i}^{m}\right)_{i=0}^{m}$ be the family of $m$-th order Chebyshev interpolation points in the interval $[-1,1]$, and let $\left(\mathcal{L}_{i}^{m}\right)_{i=0}^{m}$ be the corresponding family of Lagrange polynomials. Then

$$
\mathcal{I}^{m}[u]:=\sum_{i=0}^{m} u\left(x_{i}^{m}\right) \mathcal{L}_{i}^{m}
$$

is the corresponding one-dimensional interpolation operator. For a given interval $J:=[a, b]$ with $b-a>0$, we introduce the bijective mapping

$$
\Phi_{J}:[-1,1] \rightarrow J, \quad t \mapsto((b+a)+(b-a) t) / 2
$$


and the transformed interpolation operator

$$
\mathcal{I}_{J}^{m}[u]:=\left(\mathcal{I}^{m}\left[u \circ \Phi_{J}\right]\right) \circ \Phi_{J}^{-1} .
$$

For each $\sigma \in T$ and fixed multi-index $\mathbf{m}^{\sigma}=\left(m_{i}^{\sigma}\right)_{i=1}^{d} \in \mathbb{N}_{0}^{d}$ we set

$$
K^{\sigma}:=\left\{\nu \in \mathbb{N}_{0}^{d} \mid 0 \leq \nu_{i} \leq m_{i}^{\sigma} \text { for all } i \in\{1, \ldots, d\}\right\} .
$$

The corresponding interpolation space is

$$
\mathcal{P}^{\sigma}:=\operatorname{span}\left\{\bigotimes_{i=1}^{d} \mathcal{L}_{\nu_{i}}^{m_{i}^{\sigma}} \mid \nu \in K^{\sigma}\right\} .
$$

For $\sigma \in T$, we have $Q^{\sigma}=\prod_{i=1}^{d} J_{i}$ with intervals $J_{i}$ of positive length, and the tensor-product interpolation operator is given by

$$
\mathcal{I}^{\sigma}:=\bigotimes_{i=1}^{d} \mathcal{I}_{J_{i}}^{m_{i}^{\sigma}}
$$

For a given function $u \in C\left(Q^{\sigma}\right)$, the interpolant can be written in the form

$$
\mathcal{I}^{\sigma}[u]:=\sum_{\nu \in K^{\sigma}} u\left(x_{\nu}^{\sigma}\right) \mathcal{L}_{\nu}^{\sigma}
$$

where the interpolation points $x_{\nu}^{\sigma}$ and the corresponding Lagrange polynomials $\mathcal{L}_{\nu}^{\sigma}$ are given by

$$
x_{\nu}^{\sigma}:=\left(\Phi_{J_{i}}\left(x_{\nu_{i}}^{m_{i}^{\sigma}}\right)\right)_{i=1}^{d} \quad \text { and } \quad \mathcal{L}_{\nu}^{\sigma}:=\bigotimes_{i=1}^{d}\left(\mathcal{L}_{\nu_{i}}^{m_{i}^{\sigma}} \circ\left(\Phi_{J_{i}}\right)^{-1}\right) .
$$

Let $G \in\left\{G_{1}, G_{2}\right\}$ be a generator function (cf. 4.10) ). For each $\mathbf{b}=(\sigma, s) \in P$, we define approximations $\tilde{G}^{\mathbf{b}}: \mathbb{D} \rightarrow \mathbb{R}$ by

$$
\tilde{G}^{\mathbf{b}}:= \begin{cases}\left(\mathcal{I}^{\sigma} \otimes \mathcal{I}^{s}\right)[G] & \text { if } \mathbf{b} \text { is admissible } \\ G & \text { otherwise }\end{cases}
$$

and combine these functions by means of the hierarchical partition of unity:

$$
\tilde{G}:=\sum_{\mathbf{b} \in P} w^{\mathbf{b}} \chi^{\mathbf{b}} \tilde{G}^{\mathbf{b}} .
$$

For $\mathbf{b}=(\sigma, s) \in P_{\text {far }}$, we have defined blockwise degenerate, resp. separable, approximations

$$
\tilde{G}^{\mathbf{b}}(x, y)=\sum_{(\nu, \mu) \in K^{\mathbf{b}}} G\left(x_{\nu}^{\sigma}, x_{\mu}^{s}\right) \mathcal{L}_{\nu}^{\sigma}(x) \mathcal{L}_{\mu}^{s}(y) \quad \text { with } \quad K^{\mathbf{b}}:=K^{\sigma} \times K^{s} .
$$

Example 4.7 (Approximation properties). Let $G \in\left\{G_{1}, G_{2}\right\}$ be a generator function (cf. (4.10) ). If we have a globally uniform local error bound, i.e., an $\varepsilon \in \mathbb{R}_{>0}$ satisfying $\left\|G-\tilde{G}^{\mathbf{b}}\right\|_{\infty, Q^{\sigma} \times Q^{s}} \leq \varepsilon$ for all $\mathbf{b} \in P_{\text {far }}$, we find

$$
\begin{aligned}
\|G-\tilde{G}\|_{\infty} & =\left\|\sum_{\mathbf{b} \in P} w^{\mathbf{b}} \chi^{\mathbf{b}}\left(G-\tilde{G}^{\mathbf{b}}\right)\right\|_{\infty} \leq \sum_{\mathbf{b} \in P_{\text {far }}} w^{\mathbf{b}} \chi^{\mathbf{b}}\left\|G-\tilde{G}^{\mathbf{b}}\right\|_{\infty, \text { supp } \chi^{\mathbf{b}}} \\
& \leq \sum_{\mathbf{b}=(\sigma, s) \in P_{\text {far }}} w^{\mathbf{b}} \chi^{\mathbf{b}}\left\|G-\tilde{G}^{\mathbf{b}}\right\|_{\infty, Q^{\sigma} \times Q^{s}} \leq \sum_{\mathbf{b} \in P_{\text {far }}} w^{\mathbf{b}} \chi^{\mathbf{b}} \varepsilon=\varepsilon,
\end{aligned}
$$

so good local approximations can be combined to form a good global approximation. 
4.4. Application to kernel functions. Now we will apply the approximation scheme described in the preceding subsections to the bilinear forms given in Section 3

The relevant kernel functions are related to the generator functions by

$$
\begin{gathered}
k_{1}(x, y):=\frac{\partial}{\partial n_{y}} G_{1}(x, y), \quad k_{2}(x, y):=\left\langle n_{y}, y-x\right\rangle \frac{\partial}{\partial n_{y}} G_{1}(x, y) \\
k_{3}(x, y):=\Delta_{\Gamma, x y} G_{2}(x, y),
\end{gathered}
$$

and (3.3), (3.8) and (3.10) take the form

$$
a_{D}(u, v)=\left(\lambda-\frac{1}{2}\right)(u, v)+\int_{\Gamma} \int_{\Gamma} v(x)(u(y)-u(x)) k_{1}(x, y) d s_{y} d s_{x},
$$

$$
a_{H}(u, v)=\int_{\Gamma} \int_{\Gamma}\left\langle\overrightarrow{\operatorname{curl}}_{\Gamma} u(y), \overrightarrow{\operatorname{curl}}_{\Gamma} v(x)\right\rangle\left(k_{2}(x, y)+k_{3}(x, y)\right) d s_{y} d s_{x},
$$

$$
a_{S}(u, v)=\int_{\Gamma} \int_{\Gamma} v(x) u(y) k_{3}(x, y)+v(x)(u(y)-u(x)) k_{2}(x, y) d s_{y} d s_{x}+(\rho u, v) .
$$

Approximations of the kernel functions $k_{1}, k_{2}, k_{3}$ will be constructed by applying the surface derivatives $\partial / \partial n_{y}, \Delta_{\Gamma, x y}$, and the multiplication by $\left\langle n_{y}, x-y\right\rangle$ to the approximations of the generator functions.

We will not discuss the numerical treatment of $\left(\lambda-\frac{1}{2}\right)(u, v)$ and $(\rho u, v)$ since their representations with respect to the local boundary element basis result in sparse matrices, where the computation of the entries can be performed by simple quadrature methods. Apart from these local operators, the bilinear forms $a_{D}, a_{H}$, and $a_{S}$ can be written as sums of terms of the form

$$
a(u, v)=\int_{\Gamma \times \Gamma} v_{o p}(x) u_{o p}(y) G_{o p}(x, y) d s_{y} d s_{x},
$$

where, for $u, v \in S$, we put $u_{o p}=D_{I} u, v_{o p}=D_{I I} u, G_{o p}=\left(D_{I I I} \otimes D_{I V}\right) G$ for suitable operators

$$
D_{I}, D_{I I}, D_{I I I}, D_{I V} \in\left\{I, \frac{\partial}{\partial n_{y}},\left\langle n_{y}, y-x\right\rangle \frac{\partial}{\partial n_{y}}, \overrightarrow{\operatorname{curl}}_{i},-\nabla_{\Gamma, x}, \nabla_{\Gamma, y}^{t}\right\}
$$

depending on the underlying problem (cf. Lemma 3.1 (4.15), (4.18)).

Remark 4.8. Note that for all underlying operators the algorithmic realisation is based on the same panel-clustering algorithm, which can be realised in an abstract way independently of the concrete kernel function. This fact reduces the complexity of an implementation substantially.

Remark 4.9. Note that the term $\int_{\Gamma \times \Gamma} v(x) u(x) k_{1}(x, y) d s_{y} d s_{x}$ in (4.17) is not of the form (4.20). However, by substituting $u_{o p}(y) \leftarrow 1$ and $v_{o p}(x) \leftarrow u(x) v(x)$ in (4.20), this term can be treated in a completely analogous way.

Notation 4.10. In Section 4.3, we have introduced the approximation $\tilde{G}$ of the generator function $G$. In view of the definition of $G_{o p}$, we introduce $\tilde{G}_{o p}$ as a shorthand for $\left(D_{I I I} \otimes D_{I V}\right) \tilde{G}$. Similarly, $G_{o p}^{\mathbf{b}}$ is short for $\left(D_{I I I} \otimes D_{I V}\right)\left(\chi^{\mathbf{b}} G\right)$ and $\tilde{G}_{o p}^{\mathbf{b}}:=\left(D_{I I I} \otimes D_{I V}\right)\left(\chi^{\mathbf{b}} \tilde{G}\right)$. 
4.4.1. Approximation of a. We replace $G$ by $\tilde{G}$ in (4.20) to obtain

$$
\begin{aligned}
\tilde{a}(u, v) & :=\sum_{\mathbf{b} \in P} w^{\mathbf{b}} \int_{\Gamma} \int_{\Gamma} v_{o p}(x) u_{o p}(y) \tilde{G}_{o p}^{\mathbf{b}}(x, y) d s_{y} d s_{x} \\
= & \sum_{\mathbf{b}=(\sigma, s) \in P_{\text {near }}} w^{\mathbf{b}} \int_{\Gamma} \int_{\Gamma} v_{o p}(x) u_{o p}(y) G_{o p}^{\mathbf{b}}(x, y) d s_{y} d s_{x} \\
+ & \sum_{\mathbf{b}=(\sigma, s) \in P_{\mathrm{far}}} w^{\mathbf{b}} \sum_{(\nu, \mu) \in K^{\mathbf{b}}} G\left(x_{\nu}^{\sigma}, x_{\mu}^{s}\right)\left(\int_{\Gamma} v_{o p} D_{I I I}\left(\chi^{\sigma} \mathcal{L}_{\nu}^{\sigma}\right) d s\right) \\
& \times\left(\int_{\Gamma} u_{o p} D_{I V}\left(\chi^{s} \mathcal{L}_{\mu}^{s}\right) d s\right) .
\end{aligned}
$$

Let $\left(b_{i}\right)_{i=1}^{n}$ denote the basis of the boundary element space $S$. To obtain a more compact representation we introduce

(1) for $1 \leq i, j \leq n$, the nearfield matrix

$$
\mathbf{N}_{i, j}:=\sum_{\mathbf{b}=(\sigma, s) \in P_{\text {near }}} w^{\mathbf{b}} \int_{\Gamma} \int_{\Gamma}\left(b_{i}\right)_{o p}(x)\left(b_{j}\right)_{o p}(y) G_{o p}^{\mathbf{b}}(x, y) d s_{y} d s_{x},
$$

(2) for $\sigma \in T$ and $\nu \in K^{\sigma}$, the farfield coefficients

$$
\begin{aligned}
L_{\nu}^{\sigma}(v) & :=\int_{\Gamma} v_{o p} D_{I I I}\left(\chi^{\sigma} \mathcal{L}_{\nu}^{\sigma}\right) d s, \\
R_{\nu}^{\sigma}(u) & :=\int_{\Gamma} u_{o p} D_{I V}\left(\chi^{\sigma} \mathcal{L}_{\nu}^{\sigma}\right) d s,
\end{aligned}
$$

(3) for $\mathbf{b}=(\sigma, s) \in P_{\text {far }}$ and $(\nu, \mu) \in K^{\mathbf{b}}$ (cf. (4.14) $)$, the expansion coefficients

$$
\kappa_{\nu, \mu}^{\mathbf{b}}:=w^{\mathbf{b}} G\left(x_{\nu}^{\sigma}, x_{\mu}^{s}\right) .
$$

Then $\tilde{a}(u, v)$ can be expressed by

$$
\tilde{a}(u, v)=\mathbf{v}^{\boldsymbol{\top}} \mathbf{N u}+\sum_{\mathbf{b}=(\sigma, s) \in P_{\mathrm{far}}} \sum_{(\nu, \mu) \in K^{\mathbf{b}}} L_{\nu}^{\sigma}(v) \kappa_{\nu, \mu}^{\mathbf{b}} R_{\mu}^{s}(u) .
$$

Remark 4.11. As a consequence of the local supports of the boundary element basis functions $b_{i}$ and the cutoff functions $\chi^{\sigma}$ (cf. (4.6)), the nearfield matrix is sparse. The integral over $\Gamma \times \Gamma$ in (4.24) can be replaced by an integral over

$$
\sum_{\mathbf{b}=(k, \ell) \in P_{\text {near }}} \int_{\operatorname{supp} b_{i} \cap \operatorname{supp} \chi^{k}} \int_{\operatorname{supp} b_{j} \cap \operatorname{supp} \chi^{\ell}} \cdots,
$$

and the number $C_{\sharp}$ of blocks $\mathbf{b} \in P_{\text {near }}$ per index pair $(i, j)$, where the integration domain has positive measure, is $O(1)$.

The farfield coefficients $L_{\nu}^{\sigma}(v), R_{\nu}^{\sigma}(u)$ can be evaluated recursively by using the hierarchical structure of $T$. The details and the algorithmic realisation of a matrix-vector multiplication will be discussed in Section 5 .

4.4.2. Variable expansion order. For the efficiency of the panel-clustering method, it is essential that the farfield coefficients can be evaluated by a recurrence relation. The function systems that we have introduced in the previous sections can be written in the form

$$
\Psi_{\nu}^{\sigma}:=D_{I I I}\left(\chi^{\sigma} \mathcal{L}_{\nu}^{\sigma}\right), \quad \Phi_{\nu}^{\sigma}:=D_{I V}\left(\chi^{\sigma} \mathcal{L}_{\nu}^{\sigma}\right) \quad \forall \sigma \in T, \nu \in K^{\sigma} .
$$


where $D_{I I I}, D_{I V} \in\left\{I, \partial / \partial n,\left\langle n_{y}, y-x\right\rangle \frac{\partial}{\partial n_{y}}, \nabla_{\Gamma}, \nabla_{\Gamma}^{t}\right\}$ depend on the underlying operator (cf. (4.21)).

For the efficiency of the algorithm it is essential to evaluate the farfield coefficients $L_{\nu}^{\sigma}, R_{\mu}^{s}$ via a recursion that is based on a refinement relation of the expansion system $(4.29)$

$$
\mathcal{L}_{\nu}^{\sigma}=\sum_{\nu^{\prime} \in K^{\sigma^{\prime}}} \lambda_{\nu, \nu^{\prime}}^{\sigma, \sigma^{\prime}} \mathcal{L}_{\nu^{\prime}}^{\sigma^{\prime}} \quad \forall \nu \in K^{\sigma}, \forall \sigma \in T \text { with level }(\sigma)<L, \forall \sigma^{\prime} \in \operatorname{sons}(\sigma)
$$

with suitable shift coefficients $\lambda_{\nu, \nu^{\prime}}^{\sigma, \sigma^{\prime}}$. The functions $\mathcal{L}_{\nu}^{\sigma}$ are polynomials and, hence, relation (4.29) implies that the expansion orders are constant $K^{\sigma}=K^{\sigma^{\prime}}$ (since a polynomial cannot be represented by a polynomial of lower degree).

On the other hand, a panel-clustering method that has linear complexity requires low expansion orders on small blocks and higher expansion orders on larger blocks. This leads to a variable distribution of the expansion orders and the condition $K^{\sigma^{\prime}} \subsetneq K^{\sigma}$ for $\sigma^{\prime} \in$ sons $(\sigma)$. This is a conflict to (4.29) if we choose polynomials as the expansion system.

To overcome this problem, we will replace the Lagrange polynomials by suitable approximations and employ the refinement relation directly for their definition.

Definition 4.12. Let $\left(\Xi_{\nu}^{\sigma}\right)_{\substack{\sigma \in T \\ \nu \in K^{\sigma}}} \in\left\{\left(\Psi_{\nu}^{\sigma}\right)_{\substack{\sigma \in T \\ \nu \in K^{\sigma}}},\left(\Phi_{\nu}^{\sigma}\right)_{\substack{\sigma \in T \\ \nu \in K^{\sigma}}}\right\}$ denote one of the function systems in (4.28). For any given set of index sets $\left(K^{\sigma}\right)_{\sigma \in T}$ that satisfies

$$
K^{\sigma^{\prime}} \subset K^{\sigma} \quad \text { for all } \sigma \in T \text { with level }(\sigma)<L \text { and for all } \sigma^{\prime} \in \operatorname{sons}(\sigma) \text {, }
$$

the approximated function system $\left(\tilde{\Xi}_{\nu}^{\sigma}\right)_{\substack{\sigma \in T \\ \nu \in K^{\sigma}}}$ is given, for all $\sigma \in T$ and $\nu \in K^{\sigma}$, by

$$
\tilde{\Xi}_{\nu}^{\sigma}:= \begin{cases}\sum_{\nu}^{\sigma} \sum_{\sigma^{\prime} \in \operatorname{sons} s} \sum_{\nu_{\nu^{\prime}} \in K^{\sigma^{\prime}}}\left(\gamma^{\sigma, \sigma^{\prime}} \lambda_{\nu, \nu^{\prime}}^{\sigma, \sigma^{\prime}}\right) \tilde{\Xi}_{\nu}^{\sigma} & \text { if level }(\sigma)=L,\end{cases}
$$

with $\lambda_{\nu, \nu^{\prime}}^{\sigma, \sigma^{\prime}}=\mathcal{L}_{\nu}^{\sigma}\left(x_{\nu^{\prime}}^{\sigma^{\prime}}\right)$ and $\gamma^{\sigma, \sigma^{\prime}}$ as in (4.2).

Remark 4.13. The construction of an approximated function system that is based on a refinement relation can be applied to more general function systems as the Lagrange polynomials as well. For instance, the construction of function systems that are based on a refinement relation is very common in the context of wavelets.

Remark 4.14. If the expansion order is the same on all clusters, i.e., $K^{s}=K$ for all $s \in T$, then the approximated functions system coincides with the original one in (4.28).

Replacing the expansion systems in (4.28) by the approximated systems

$$
\left(\tilde{\Psi}_{\nu}^{\sigma}\right)_{\substack{\sigma \in T^{\sigma} \\ \nu \in K^{\sigma}}}, \quad\left(\tilde{\Phi}_{\nu}^{\sigma}\right)_{\substack{\sigma \in T \\ \nu \in K^{\sigma}}}
$$

leads to approximated farfield coefficients $\tilde{L}_{\nu}^{\sigma}, \tilde{R}_{\nu}^{\sigma}$ in (4.25).

Lemma 4.15. Let

$$
\tilde{L}_{\nu}^{\sigma}(v):=\int_{\Gamma} v_{o p} \tilde{\Psi}_{\nu}^{\sigma} d s, \quad \tilde{R}_{\mu}^{s}(u):=\int_{\Gamma} u_{o p} \tilde{\Phi}_{\nu}^{\sigma} d s
$$


Then the recursion for the farfield coefficients

$$
\tilde{L}_{\nu}^{\sigma}(v)=\sum_{\sigma^{\prime} \in \operatorname{sons}(\sigma)} \sum_{\nu^{\prime} \in K^{\sigma^{\prime}}} \gamma^{\sigma, \sigma^{\prime}} \lambda_{\nu, \nu^{\prime}}^{\sigma, \sigma^{\prime}} \tilde{L}_{\nu^{\prime}}^{\sigma^{\prime}}(v)
$$

holds for all $\sigma \in T$ with level $(\sigma)<L$ and $\nu \in K^{\sigma}$. The coefficients $\tilde{R}_{\mu}^{s}$ satisfy the same recurrence.

Finally, we will specify the index sets $K^{\sigma}$ for the definition of the approximated expansion system $\left(\tilde{\mathcal{L}}_{\nu}^{\sigma}\right)_{\substack{\sigma \in T \\ \nu \in K^{\sigma}}}$. Recall the definition of the axis-parallel cubes $Q^{\sigma}=$ $J_{1}^{\sigma} \times J_{2}^{\sigma} \times J_{3}^{\sigma}$ as in (4.5) and (4.11). The index sets are of the form $K^{\sigma}:=\mathbb{N}_{m_{1}^{\sigma}} \times$ $\mathbb{N}_{m_{2}^{\sigma}} \times \mathbb{N}_{m_{3}^{\sigma}}$, where $\mathbb{N}_{k}:=\{0,1, \ldots, k\}$ and the order distributions $\mathbf{m}^{\sigma}=\left(m_{j}^{\sigma}\right)_{j=1}^{3}$ depend on three parameters $\alpha, \beta \in \mathbb{N}_{0}, 0<\bar{q}<1$. For $1 \leq j \leq 3$, they are defined by

(4.33a) $m_{j}^{\sigma}:= \begin{cases}\beta & \text { if level }(\sigma)=L, \\ \min _{\sigma^{\prime} \in \operatorname{sons}(\sigma)}\left\{m_{j}^{\sigma^{\prime}}+\alpha_{j}^{\sigma^{\prime}, \sigma}\right\} & \text { if level }(\sigma)<L,\end{cases}$

with

$$
\alpha_{j}^{\sigma^{\prime}, \sigma}:= \begin{cases}\alpha & \text { if }\left|J_{j}^{\sigma^{\prime}}\right| /\left|J_{j}^{\sigma}\right| \leq \bar{q} \\ 0 & \text { otherwise }\end{cases}
$$

The definition of $\alpha_{j}^{\sigma^{\prime}, \sigma}$ reflects the fact that an increase of the approximation order is necessary only if the lengths $\left|J_{j}^{\sigma^{\prime}}\right|$ that are related to the sons $\sigma^{\prime} \in \operatorname{sons}(\sigma)$ are sufficiently smaller than the corresponding length $\left|J_{j}^{\sigma}\right|$ of the father.

4.4.3. Panel-clustering with variable expansion order. Let $\alpha, \beta \in \mathbb{N}_{0}$ and $0<\bar{q} \leq 1$ be fixed. Recall the definition of the index sets $K^{\sigma}$ as in (4.33) and define, for $\mathbf{b}=(\sigma, s) \in P_{\text {far }}$, the associated index sets $K^{\mathbf{b}}:=K^{\sigma} \times K^{s}$. The approximation order is given by $\mathbf{m}^{\mathbf{b}}:=\left(\mathbf{m}^{\sigma}, \mathbf{m}^{s}\right)$. Let the farfield coefficients $\tilde{L}_{\nu}^{\sigma}, \tilde{R}_{\nu}^{\sigma}$ be defined by (4.31). Then, the panel-clustering approximation with variable order of the bilinear form $a(\cdot, \cdot)$ is given by

$$
\tilde{a}(u, v)=\mathbf{v}^{\boldsymbol{\top}} \mathbf{N u}+\sum_{\mathbf{b}=(\sigma, s) \in P_{\mathrm{far}}} \sum_{(\nu, \mu) \in K^{\mathbf{b}}} \tilde{L}_{\nu}^{\sigma}(v) \kappa_{\nu, \mu}^{\mathbf{b}} \tilde{R}_{\mu}^{s}(u) .
$$

\section{Algorithm}

In this section, we will explain the efficient algorithmic realisation of a matrixvector multiplication, i.e., $\left(\tilde{a}\left(b_{i}, u\right)\right)_{i=1}^{n}$. The farfield coefficients $\tilde{L}_{\nu}^{\sigma}, \tilde{R}_{\mu}^{s}$ are of the abstract form (4.31), and the expansion coefficients $\kappa_{\nu, \mu}^{\mathrm{b}}$ are the evaluation of the generator function at the interpolation nodes:

$$
\kappa_{\nu, \mu}^{\mathbf{b}}:=w^{\mathbf{b}} G\left(x_{\nu}^{\sigma}, x_{\mu}^{s}\right) \quad \forall \mathbf{b}=(\sigma, s) \in P_{\text {far }}, \quad \forall(\nu, \mu) \in K^{\mathbf{b}}:=K^{\sigma} \times K^{s},
$$

where the choice of $G \in\left\{G_{1}, G_{2}\right\}$ depends on the underlying operator. 
5.1. Numerical quadrature. The computation of the nearfield matrix $\mathbf{N}$ involves the evaluation of singular and nearly singular surface integrals.

The nearfield integrals are approximated by the quadrature technique introduced in [28, [17, [8]. We distinguish between the proper singular case, where the kernel function is singular in the domain of integration and the nearly singular case, where the kernel function is analytic but its derivatives blow up as the distance of the panels tends to zero.

For the proper singular integrals, regularising coordinate transforms (relative coordinates) are introduced rendering the integrands analytic (cf. [28, [17, [8], [27]). Then, properly scaled tensor Gauß quadrature rules are employed for the numerical approximation.

The nearly singular integrals are directly treated by properly scaled tensor Gauß rules where the order is chosen according to the error estimates. We do not recapitulate the quadrature formulae but refer to [8], 27] for a compact reference.

The error analysis in Section [6 will show that these quadrature techniques require only $O(1)$ kernel evaluations per matrix entry to obtain an approximation $\tilde{\mathbf{N}}$ of the nearfield matrix such that the corresponding perturbed Galerkin solution converges with the same rate as the exact Galerkin solution.

5.2. Setup phase of the panel-clustering algorithm. In this section, we will develop an efficient algorithm to evaluate the second term in (4.34), namely the farfield part

$$
\tilde{a}_{\mathrm{far}}(u, v):=\sum_{\mathbf{b}=(\sigma, s) \in P_{\mathrm{far}}} \sum_{(\nu, \mu) \in K^{\mathbf{b}}} \kappa_{\nu, \mu}^{\mathbf{b}} \tilde{L}_{\nu}^{\sigma}(v) \tilde{R}_{\mu}^{s}(u) .
$$

The farfield coefficients can be represented by the farfield coefficient matrices $\mathbf{L}^{\sigma}=\left(\mathbf{L}_{i, \nu}^{\sigma}\right)_{\substack{1 \leq i \leq n \\ \nu \in K^{\sigma}}}, \mathbf{R}^{\sigma}=\left(\mathbf{R}_{i, \nu}^{\sigma}\right)_{\substack{1 \leq i \leq n \\ \nu \in K^{\sigma}}}$, which are defined for all clusters $\sigma \in T$ and all $1 \leq i \leq n$ by

$$
\mathbf{L}_{i, \nu}^{\sigma}:=\int_{\Gamma}\left(b_{i}\right)_{o p} \tilde{\Psi}_{\nu}^{\sigma} d s, \quad \mathbf{R}_{i, \nu}^{\sigma}:=\int_{\Gamma}\left(b_{i}\right)_{o p} \tilde{\Phi}_{\nu}^{\sigma} d s .
$$

The expansion coefficients are the entries of the interaction matrices

$$
\mathbf{S}^{\mathbf{b}}=\left(\kappa_{\nu, \mu}^{\mathbf{b}}\right)_{\nu, \mu \in K^{\mathbf{b}}}
$$

with the expansion coefficients as in (5.2). Using these matrices, the bilinear form $\tilde{a}_{\text {far }}(\cdot, \cdot)$ in (5.2) equals

$\mathbf{v}^{\boldsymbol{\top}}\left(\sum_{\mathbf{b}=(\sigma, s) \in P_{\mathrm{far}}} \mathbf{L}^{\sigma} \mathbf{S}^{\mathbf{b}}\left(\mathbf{R}^{s}\right)^{\boldsymbol{\top}}\right) \mathbf{u}:=\sum_{i, j=1}^{n} \mathbf{v}_{i}\left(\sum_{\mathbf{b}=(\sigma, s) \in P_{\mathrm{far}}} \sum_{(\nu, \mu) \in K^{\mathbf{b}}} \mathbf{S}_{\nu, \mu}^{\mathbf{b}} \mathbf{L}_{i, \nu}^{\sigma} \mathbf{R}_{j, \mu}^{s}\right) \mathbf{u}_{j}$.

In the next step, we will use the recurrence relation (4.32) to avoid the storage of the farfield coefficient matrices $\mathbf{L}^{\sigma}, \mathbf{R}^{\sigma}$. Instead we define, for all $\sigma \in T$ with level $(\sigma)<L$ and $\sigma^{\prime} \in$ sons $(\sigma)$ the shift matrix $\mathbf{B}^{\sigma^{\prime}, \sigma}=\left(\mathbf{B}_{\nu^{\prime}, \nu}^{\sigma^{\prime}, \sigma}\right)_{\substack{\nu \in K^{\sigma} \\ \nu^{\prime} \in K^{\sigma^{\prime}}}}$ by

$$
\mathbf{B}_{\nu^{\prime}, \nu}^{\sigma^{\prime}, \sigma}:=\gamma^{\sigma, \sigma^{\prime}} \lambda_{\nu, \nu^{\prime}}^{\sigma, \sigma^{\prime}} \quad \text { with } \quad \gamma^{\sigma, \sigma^{\prime}} \text { as in (4.2) and } \lambda_{\nu, \nu^{\prime}}^{\sigma, \sigma^{\prime}} \text { as in (4.29). }
$$


The relation (4.32) implies that

$$
\mathbf{R}^{\sigma}=\sum_{\sigma^{\prime} \in \operatorname{sons}(\sigma)} \mathbf{R}^{\sigma^{\prime}} \mathbf{B}^{\sigma^{\prime}, \sigma}:=\left(\sum_{\sigma^{\prime} \in \operatorname{sons}(\sigma)} \sum_{\nu^{\prime} \in K^{\sigma^{\prime}}} \mathbf{R}_{i, \nu^{\prime}}^{\sigma^{\prime}} \mathbf{B}_{\nu^{\prime}, \nu}^{\sigma^{\prime}, \sigma}\right)_{\substack{1 \leq i \leq n \\ \nu \in K^{\sigma}}},
$$

and the same relation holds for the farfield coefficient matrices $\mathbf{L}^{\sigma}$.

In summary, the fast evaluation of the bilinear form $\tilde{a}_{\mathrm{far}}(\cdot, \cdot)$ in (5.2) requires a setup phase that consists of the following steps.

(1) Compute and store the interaction matrices $\mathbf{S}^{\mathbf{b}}$ for each farfield block $\mathbf{b} \in$ $P_{\text {far. }}$.

(2) For all $\sigma \in T$ with level $(\sigma)<L$ and all $\sigma^{\prime} \in \operatorname{sons}(\sigma)$ compute and store the shift matrices $\mathbf{B}^{\sigma^{\prime}, \sigma}$.

(3) For all $\sigma \in T$ with level $(\sigma)=L$ compute and store the basis farfield coefficient matrix

$$
\mathbf{L}_{i, \nu}^{\sigma}:= \begin{cases}\int_{\Gamma}\left(b_{i}\right)_{o p} \Psi_{\nu}^{\sigma} d s & \forall i \in\{1,2, \ldots, n\}:\left|\operatorname{supp} b_{i} \cap \operatorname{supp} \chi^{\sigma}\right|>0 \\ 0 & \text { otherwise, }\end{cases}
$$

in a sparse format. Proceed with the basis farfield coefficient matrix $\mathbf{R}_{i, \nu}^{\sigma}$ in the same manner.

5.3. Fast matrix-vector multiplication. Let $\mathbf{K}_{\mathrm{far}}$ denote the representation of the bilinear form $\tilde{a}_{\mathrm{far}}(\cdot, \cdot)$ with respect to the basis $\left(b_{i}\right)_{i=1}^{n}$ of the boundary element space. Recall the convention (2.6) relating a coefficient vector $\mathbf{u}=\left(u_{i}\right)_{i=1}^{n} \in \mathbb{R}^{n}$ with the corresponding boundary element function $u$.

In this section, we will present an algorithm for the fast evaluation of $\mathbf{K}_{\mathrm{far}} \mathbf{u}=$ $\left(\tilde{a}_{\text {far }}\left(b_{i}, u\right)\right)_{i=1}^{n}$. For $\sigma \in T$, let

$$
P_{\text {far }}(\sigma):=\left\{s \in T:(\sigma, s) \in P_{\text {far }}\right\} .
$$

For a given vector $\mathbf{u} \in \mathbb{R}^{n}$, we define

$$
\mathbf{r}^{s}:=\left(\mathbf{r}_{\nu}^{s}\right)_{\nu \in K^{s}}:=\left(\mathbf{R}^{s}\right)^{\top} \mathbf{u} \text { and } \quad \mathbf{s}^{\sigma}:=\sum_{s \in P_{\mathrm{far}}(\sigma)} \mathbf{S}^{\sigma, s} \mathbf{r}^{s}
$$

and find

$$
\mathbf{y}:=\mathbf{K}_{\mathrm{far}} \mathbf{u}=\sum_{\mathbf{b}=(\sigma, s) \in P_{\mathrm{far}}} \mathbf{L}^{\sigma} \mathbf{S}^{\mathbf{b}}\left(\mathbf{R}^{s}\right)^{\boldsymbol{\top}} \mathbf{u}=\sum_{\mathbf{b}=(\sigma, s) \in P_{\mathrm{far}}} \mathbf{L}^{\sigma} \mathbf{S}^{\mathbf{b}} \mathbf{r}^{s}=\sum_{\sigma \in T} \mathbf{L}^{\sigma} \mathbf{s}^{\sigma}
$$

For $\sigma \in T$ with level $(\sigma)<L$, the computation of $\mathbf{r}^{\sigma}$ can be written in the form

$$
\mathbf{r}^{\sigma}=\left(\mathbf{R}^{\sigma}\right)^{\top} \mathbf{u}=\sum_{\sigma^{\prime} \in \operatorname{sons}(\sigma)}\left(\mathbf{B}^{\sigma^{\prime}, \sigma}\right)^{\top}\left(\mathbf{R}^{\sigma^{\prime}}\right)^{\top} \mathbf{u}=\sum_{\sigma^{\prime} \in \operatorname{sons}(\sigma)}\left(\mathbf{B}^{\sigma^{\prime}, \sigma}\right)^{\top} \mathbf{r}^{\sigma^{\prime}},
$$

so we can compute $\mathbf{r}=\left(\mathbf{r}^{\sigma}\right)_{\sigma \in T}$ by the recursive procedure forward which starts from the coarsest cluster level $T_{0} \subset T$. The procedure is called by

for $\sigma_{0} \in T_{0} \quad$ do $\quad$ forward $\left(\mathbf{u}, \sigma_{0}, \mathbf{r}\right)$;

and defined by

procedure forward $(\mathbf{u}, \sigma, \mathbf{r})$;

begin

if level $(\sigma)=L$ then $\mathbf{r}^{\sigma}:=\mathbf{R}^{\sigma} \mathbf{u}$ 
else begin

$$
\begin{aligned}
& \mathbf{r}^{\sigma}:=0 \\
& \text { for } \sigma^{\prime} \in \operatorname{sons}(\sigma) \text { begin }
\end{aligned}
$$

\section{end end end;}

$\operatorname{forward}\left(\mathbf{u}, \sigma^{\prime}, \mathbf{r}\right) ; \mathbf{r}^{\sigma}:=\mathbf{r}^{\sigma}+\left(\mathbf{B}^{\sigma^{\prime}, \sigma}\right)^{\top} \mathbf{r}^{\sigma^{\prime}}$

By the similar procedure backward we can use the matrices $\mathbf{B}^{\sigma^{\prime}, \sigma}$ to compute $\mathbf{y}$ from the coefficients $\mathbf{s}^{\sigma}$. The procedure is called by

$$
\text { for } \quad \sigma_{0} \in T_{0} \quad \text { do } \quad \operatorname{backward}\left(\mathbf{y}, \sigma_{0}, \mathbf{s}\right) \text {; }
$$

and defined by

$$
\begin{aligned}
& \text { procedure backward }(\mathbf{y}, \sigma, \mathbf{s}) \\
& \text { begin } \\
& \text { if level }(\sigma)=L \text { then } \\
& \mathbf{y}:=\mathbf{y}+\mathbf{L}^{\sigma} \mathbf{s}^{\sigma} \\
& \text { else for } \sigma^{\prime} \in \operatorname{sons}(\sigma) \text { do begin } \\
& \mathbf{s}^{\sigma^{\prime}}:=\mathbf{s}^{\sigma^{\prime}}+\mathbf{B}^{\sigma^{\prime}, \sigma} \mathbf{s}^{\sigma} ; \text { backward }\left(\mathbf{y}, \sigma^{\prime}, \mathbf{s}\right) ;
\end{aligned}
$$

end end;

We can combine the forward and backward transformation in order to devise a fast algorithm for the matrix-vector multiplication. Recall the definition of $P_{\text {far }}(\sigma)$ as in (5.4).

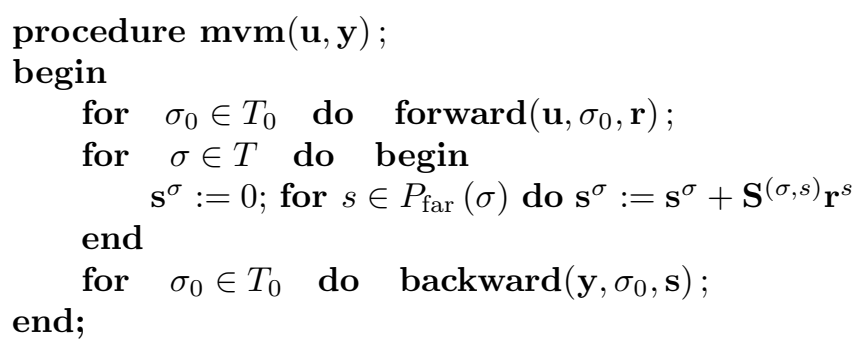

\section{ERROR ANALYSIS}

In this section, we develop the error analysis for the variable order panel-clustering method applied to the alternative representations of boundary integral operators. As a result we obtain the distribution of the expansion order on different cluster levels and the quadrature orders for the nearfield part.

We restrict here to low-order boundary element discretisations with quasi-uniform and shape regular meshes (cf. Definitions 6.16 and 6.17). For higher-order boundary elements, the use of graded meshes that are neither quasi-uniform nor shape regular becomes important. The panel-clustering method for those types of meshes will be discussed in [10].

6.1. Interpolation error estimate. Let $G \in\left\{G_{1}, G_{2}\right\}$. $G$ is asymptotically smooth, i.e., we have

$$
\left|\partial_{x}^{\nu} \partial_{y}^{\mu} G(x, y)\right| \leq C_{\text {asymp }}(\nu+\mu) ! c_{0}^{|\nu+\mu|}\|x-y\|^{-g-|\nu+\mu|}
$$

for constants $C_{\text {asymp }}, c_{0} \in \mathbb{R}_{>0}$. This estimate follows from Cauchy's integral formula (see, e.g., [16, Appendix B], [25, Lemma 4.24]). The order of singularity $g$ equals 1 for $G=G_{1}$ and is -1 for $G=G_{2}$. 
For each $m \in \mathbb{N}$, let $\Lambda_{m} \in \mathbb{R}_{\geq 1}$ be the smallest constant such that

$$
\left\|\mathcal{I}^{m} u\right\|_{\infty,[-1,1]} \leq \Lambda_{m}\|u\|_{\infty,[-1,1]}
$$

for all $u \in C([-1,1])$, i.e., $\Lambda_{m}$ is the Lebesgue constant for the $m$ th-order interpolation operator. We assume that there are constants $C_{\lambda}, \lambda \in \mathbb{R}_{>0}$ satisfying

$$
\Lambda_{m} \leq C_{\lambda}(m+1)^{\lambda}
$$

for all $m \in \mathbb{N}$, i.e., that the interpolation scheme is stable (in the case of Chebyshev interpolation, we have $C_{\lambda}=\lambda=1$; cf. [23]).

In [2, Corollary 3.8], the fundamental estimate

$$
\left\|u-\mathcal{I}_{J^{\prime}}^{k} u\right\|_{\infty, J^{\prime}} \leq C\left(1+\Lambda_{k}\right)(4 p)^{p-k-1}\left(\frac{\left|J^{\prime}\right|}{|J|}\right)^{k+1}\|u\|_{\infty, J}
$$

has been proven for polynomials $u \in \mathcal{P}^{p}$ and intervals $J^{\prime} \subseteq J$. Since our kernel approximations are constructed as derivatives of variable-order interpolants of the generating functions $G_{1}$ and $G_{2}$, we need to introduce derivatives into the above estimate.

Lemma 6.1. Let $J^{\prime} \subseteq J$ be intervals. For $p, k, l \in \mathbb{N}_{0}$ with $l \leq k \leq p$ and for all $u \in \mathcal{P}^{p}$, we have

$$
\left\|u^{(l)}-\left(\mathcal{I}_{J^{\prime}}^{k} u\right)^{(l)}\right\|_{\infty, J^{\prime}} \leq \frac{C}{l !}\left(1+\Lambda_{k}\right) p^{2 l}(4 p)^{p-k-1}\left(\frac{\left|J^{\prime}\right|}{|J|}\right)^{k-l+1}\left\|u^{(l)}\right\|_{\infty, J} .
$$

Proof. Let $z \in J$ be the midpoint of $J$ and let $\bar{u}$ be the $l$-th order Taylor expansion of $u$ in the point $z$. Then we have

$$
\|u-\bar{u}\|_{\infty, J} \leq \frac{1}{l !}\left(\frac{|J|}{2}\right)^{l}\left\|u^{(l)}\right\|_{\infty, J} .
$$

We apply Markov's inequality (cf., e.g., [6, Thm. 4.1.4])

$$
\left\|u^{(l)}\right\|_{\infty, J^{\prime}} \leq\left(\frac{2}{\left|J^{\prime}\right|}\right)^{l}\left[\begin{array}{l}
p \\
l
\end{array}\right]^{2}\|u\|_{\infty, J^{\prime}} \quad \text { with } \quad\left[\begin{array}{l}
p \\
l
\end{array}\right]=p ! /(p-l) !
$$

in order to find

$$
\begin{aligned}
\left\|\left(u-\mathcal{I}_{J^{\prime}}^{k} u\right)^{(l)}\right\|_{\infty, J^{\prime}} & \stackrel{\sqrt[6.60]{\leq}}{\leq}\left(\frac{2}{\left|J^{\prime}\right|}\right)^{l}\left[\begin{array}{l}
p \\
l
\end{array}\right]^{2}\left\|u-\mathcal{I}_{J^{\prime}}^{k} u\right\|_{\infty, J^{\prime}} \\
& =\left(\frac{2}{\left|J^{\prime}\right|}\right)^{l}\left[\begin{array}{l}
p \\
l
\end{array}\right]^{2}\left\|(u-\bar{u})-\mathcal{I}_{J^{\prime}}^{k}(u-\bar{u})\right\|_{\infty, J^{\prime}} \\
& \stackrel{(6.4)}{\leq} C\left(1+\Lambda_{k}\right)(4 p)^{p-k-1} 2^{l} \frac{\left|J^{\prime}\right|^{k-l+1}}{|J|^{k+1}}\left[\begin{array}{c}
p \\
l
\end{array}\right]^{2}\|u-\bar{u}\|_{\infty, J} \\
& \stackrel{(6.5)}{\leq} C\left(1+\Lambda_{k}\right)(4 p)^{p-k-1}\left(\frac{\left|J^{\prime}\right|}{|J|}\right)^{k-l+1}\left[\begin{array}{c}
p \\
l
\end{array}\right]^{2} \frac{1}{l !}\left\|u^{(l)}\right\|_{\infty, J} .
\end{aligned}
$$

We conclude the proof by observing

$$
\left[\begin{array}{l}
p \\
l
\end{array}\right]=\frac{p !}{(p-l) !}=\prod_{i=0}^{l-1}(p-i) \leq p^{l} .
$$


By similar techniques, we find

$$
\left\|\left(\mathcal{I}_{J}^{k} w\right)^{(l)}\right\|_{\infty, J} \leq \Lambda_{k} k^{2 l}\left\|w^{(l)}\right\|_{\infty, J},
$$

for all $k, l \in \mathbb{N}$ with $l \leq k$ and all $w \in C^{l}([-1,1])$.

Using (6.3), these estimates take the form

$$
\begin{aligned}
\left\|u^{(l)}-\left(\mathcal{I}_{J^{\prime}}^{k} u\right)^{(l)}\right\|_{\infty, J^{\prime}} & \leq \frac{C^{\prime}}{l !}(p+1)^{\lambda+2 l}(4 p)^{p-k-1}\left(\frac{\left|J^{\prime}\right|}{|J|}\right)^{k-l+1}\left\|u^{(l)}\right\|_{\infty, J}, \\
\left\|\left(\mathcal{I}_{J}^{k} w\right)^{(l)}\right\|_{\infty, J} & \leq C_{\lambda}(k+1)^{\lambda+2 l}\left\|w^{(l)}\right\|_{\infty, J}
\end{aligned}
$$

for $u \in \mathcal{P}^{p}, w \in C^{l}([-1,1])$ and a constant $C^{\prime} \in \mathbb{R}_{>0}$. This means that the only difference between the original estimates for the interpolants and our estimates for their derivatives are the increased stability constants and the decreased approximation order.

Next, we will derive an approximation property for analytic functions.

Lemma 6.2. Let $J$ be a closed interval and $u \in C^{\infty}(J)$ such that, for some $C_{u}$, $\gamma_{u}>0$, it follows that

$$
\left\|u^{(n)}\right\|_{\infty, J} \leq C_{u} \gamma_{u}^{n} n ! \quad \forall n \in \mathbb{N}
$$

Then

$$
\inf _{p \in \mathcal{P}^{k}}\|u-p\|_{\infty, J} \leq C C_{u}\left(1+\frac{1}{c_{0}}\right)^{-k-1} \quad \text { with } \quad c_{0}:=\gamma_{u}|J|,
$$

where $C$ only depends on $c_{0}$.

Proof. The proof is a direct consequence of [2, Lemma 3.13].

Definition 6.3 (Cluster sequence). Let $\sigma \in T$, $\ell:=\operatorname{level}(\sigma)$. A sequence $\vec{\sigma}=$ $\left(\sigma_{i}\right)_{i=\ell}^{L} \subset T$ satisfying $\sigma_{\ell}=\sigma$ and $\sigma_{i+1} \in \operatorname{sons}\left(\sigma_{i}\right)$ for all $i \in\{\ell, \ldots, L-1\}$ is a cluster sequence corresponding to $\sigma$. The set of all such cluster sequences is denoted by $\overrightarrow{\mathfrak{C}}^{\sigma}$.

For each cluster sequence $\vec{\sigma}=\left(\sigma_{i}\right)_{i=\ell}^{L} \in \overrightarrow{\mathfrak{C}}^{\sigma}$ and $\ell \leq k \leq L$, the composite interpolation operator is given by

$$
\mathcal{I}_{k}^{\vec{\sigma}}:=\mathcal{I}^{\sigma_{L}} \circ \mathcal{I}^{\sigma_{L-1}} \ldots \circ \mathcal{I}^{\sigma_{k}} .
$$

If $k=\ell$, we skip the index $k$ and write $\mathcal{I}^{\vec{\sigma}}$ instead of $\mathcal{I}_{\ell}^{\vec{\sigma}}$. The relation of the iterated interpolation operator on cluster sequences and the approximated function systems (cf. (4.30) is given by

$$
\tilde{\Xi}_{\nu}^{\sigma}=\sum_{\vec{\sigma} \in \overrightarrow{\mathfrak{C}}^{\sigma}} \gamma^{\vec{\sigma}} D\left(\chi^{\sigma_{L}} \mathcal{I}^{\vec{\sigma}}\left(L_{\nu}^{\sigma}\right)\right)
$$

where, for $\vec{\sigma}=\left(\sigma_{i}\right)_{i=\ell}^{L}$, we set $\gamma^{\vec{\sigma}}:=\prod_{i=\ell}^{L-1} \gamma^{\sigma_{i}, \sigma_{i+1}}$. In (6.8) $), \sigma_{L}$ is the last component of the sequence $\vec{\sigma}, \chi^{\sigma_{L}}$ is the corresponding cutoff function. $L_{\nu}^{\sigma}$ are the Lagrange polynomials as in (4.12), and $D \in\left\{D_{I I I}, D_{I V}\right\}$ depends on the choice of $\Xi_{\nu}^{\sigma}($ cf. (4.28) $)$. 
Due to (4.33) and the resulting anisotropic distribution of the interpolation orders, the number of iterated interpolations varies among the three spatial dimensions, so we introduce upper and lower bounds for these numbers by

$$
\begin{aligned}
& \bar{n}^{\vec{\sigma}}:=\max \left\{\#\left\{i \in\{\ell, \ldots, L-1\}: m_{j}^{\sigma_{i}}<m_{j}^{\sigma_{i+1}}\right\}: j \in\{1,2,3\}\right\}, \\
& \underline{n}^{\vec{\sigma}}:=\min \left\{\#\left\{i \in\{\ell, \ldots, L-1\}: m_{j}^{\sigma_{i}}<m_{j}^{\sigma_{i+1}}\right\}: j \in\{1,2,3\}\right\} .
\end{aligned}
$$

Next, we will express the variable order approximation of the kernel functions by using the iterated interpolation operator and start with the approximation of the generator function. We have for the farfield approximation,

$$
G_{\text {far }}:=\sum_{\mathbf{b} \in P_{\text {far }}} w^{\mathbf{b}} \underbrace{\sum_{\overrightarrow{\mathbf{b}} \in \overrightarrow{\mathfrak{c}} \mathbf{b}} \gamma^{\overrightarrow{\mathbf{b}}} \chi^{\overrightarrow{\mathbf{b}}} G}_{=: G_{\text {far }}^{\mathbf{b}}} \approx \tilde{G}_{\text {far }}=\sum_{\mathbf{b} \in P_{\text {far }}} w^{\mathbf{b}} \underbrace{\sum_{\overrightarrow{\mathbf{b}} \in \overrightarrow{\mathfrak{c}} \mathbf{b}} \gamma^{\overrightarrow{\mathbf{b}}} \chi^{\overrightarrow{\mathbf{b}}} \mathcal{I}^{\overrightarrow{\mathbf{b}}} G}_{=: \tilde{G}_{\text {far }}^{\mathbf{b}}},
$$

where, for $\mathbf{b}=(\sigma, s) \in P_{\text {far }}$, we employ the notation $\overrightarrow{\mathfrak{C}}^{\mathbf{b}}:=\overrightarrow{\mathfrak{C}}^{\sigma} \times \overrightarrow{\mathfrak{C}}^{s}$ and, for $\vec{\sigma}=\left(\sigma_{i}\right)_{i=\ell}^{L}, \vec{s}=\left(s_{i}\right)_{i=\ell}^{L}$ and a corresponding block sequence $\overrightarrow{\mathbf{b}}=(\vec{\sigma}, \vec{s}) \in \overrightarrow{\mathfrak{C}^{\mathbf{b}}}$, we put

$$
\chi^{\overrightarrow{\mathbf{b}}}:=\chi^{\sigma_{L}} \otimes \chi^{s_{L}}, \quad \gamma^{\overrightarrow{\mathbf{b}}}:=\gamma^{\vec{\sigma}} \gamma^{\vec{s}}, \quad \text { and } \quad \mathcal{I}_{k}^{\overrightarrow{\mathbf{b}}}:=\mathcal{I}_{k}^{\vec{\sigma}} \otimes \mathcal{I}_{k}^{\vec{s}} .
$$

Again, for $k=\ell$, we skip the index $L$ in $\mathcal{I}_{k}^{\overrightarrow{\mathbf{b}}}$.

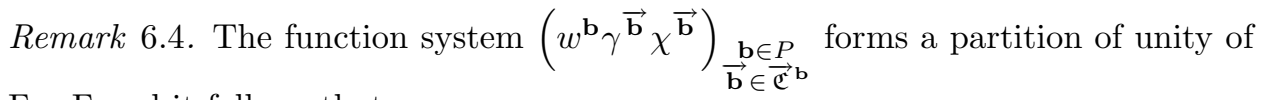
$\Gamma \times \Gamma$ and it follows that

$$
\chi^{\mathbf{b}}=\sum_{\overrightarrow{\mathbf{b}} \in \overrightarrow{\vec{c}^{\mathbf{b}}}} \gamma^{\overrightarrow{\mathbf{b}}} \chi^{\overrightarrow{\mathbf{b}}}
$$

Farfield approximations to the kernel function $k=G_{o p}$ (cf. (4.20)) are derived by applying the operator $D_{I I I} \otimes D_{I V}$ to $\tilde{G}_{\text {far }}$. Recalling (6.9), we get

$$
\sum_{\mathbf{b} \in P_{\text {far }}} \tilde{k}^{\mathbf{b}}(x, y):=\sum_{\mathbf{b} \in P_{\text {far }}} w^{\mathbf{b}} \sum_{\overrightarrow{\mathbf{b}} \in \overrightarrow{\mathfrak{C}} \mathbf{b}}\left(\gamma^{\overrightarrow{\mathbf{b}}} \chi^{\overrightarrow{\mathbf{b}}} \mathcal{I}^{\overrightarrow{\mathbf{b}}} G\right)_{o p}(x, y) .
$$

The farfield part of the exact kernel is defined by replacing the term $\left(\chi^{\overrightarrow{\mathbf{b}}} \mathcal{I}^{\overrightarrow{\mathbf{b}}} G\right)_{o p}$ in (6.11) by $\left(\chi^{\overrightarrow{\mathbf{b}}} G\right)_{o p}$.

Let us consider an admissible block $(\sigma, s) \in P_{\text {far. }}$ Let $\vec{\sigma}=\left(\sigma_{i}\right)_{i=\ell}^{L}$ and $\vec{s}=$ $\left(s_{i}\right)_{i=\ell}^{L}$ be cluster sequences corresponding to $\sigma$ and $s$. Let the parameter $\beta \in \mathbb{N}_{0}$ (cf. 4.33) satisfy $\beta>1$. Let $\bar{n}:=\max \left\{\bar{n}^{\vec{\sigma}}, \bar{n}^{\vec{s}}\right\}$ and $\underline{n}:=\min \left\{\underline{n}^{\vec{\sigma}}, \underline{n}^{\vec{s}}\right\}$. Using our results on differentiated interpolants, we can generalize [2, Theorem 4.3] in order to find a polynomial $C$ and constants $\bar{\alpha}>0$ and $0 \leq c_{2}<1$ satisfying

$$
\left\|\partial_{x}^{\nu} \partial_{y}^{\mu}\left(G-\mathcal{I}^{\overrightarrow{\mathbf{b}}} G\right)\right\|_{\infty, \operatorname{supp}} \chi^{\overrightarrow{\mathbf{b}}} \leq C(\alpha, \beta-\delta, \bar{n}) \operatorname{dist}\left(Q^{\sigma}, Q^{s}\right)^{-g-|\nu+\mu|} c_{2}^{\beta+\min \{(\beta \bar{\alpha}, \alpha\} \underline{n}},
$$

for all multi-indices $\nu, \mu \in \mathbb{N}_{0}^{3}$ with $|\nu|,|\mu| \in\{0,1\}$.

Under reasonable assumptions the right-hand side in (6.12) can be expressed in terms of the levels $\ell, L$. 
Assumption 6.5. All blocks $\mathbf{b}=(\sigma, s) \in P$ consist of clusters of the same level $\sigma, s \in T_{\ell}$ for some $0 \leq \ell \leq L$.

There exists a constant $\gamma>0$ depending only on $\bar{q}$ (in (4.33b) and on the partition of unity such that

$$
\gamma(L-\ell) \leq \underline{n}^{\vec{\sigma}} \quad \forall \sigma \in T, \vec{\sigma} \in \overrightarrow{\mathfrak{C}}^{\sigma}, \ell=\operatorname{level}(\sigma) .
$$

In view of (6.12), (6.13) we introduce

$$
m_{\ell}:=\beta+\underline{\alpha}(L-\ell) \quad \text { with } \quad \underline{\alpha}:=\min \{\beta \bar{\alpha}, \alpha\} \gamma .
$$

Lemma 6.6. Let Assumption 6.5 and (4.6) be valid and $g+|\mu+\nu| \geq 0$.

Then, the right-hand side in (6.12) can be estimated from above and we obtain the estimate

$$
\left\|\partial_{x}^{\nu} \partial_{y}^{\mu}\left(G-\mathcal{I}^{\overrightarrow{\mathbf{b}}} G\right)\right\|_{\infty, \operatorname{supp} \chi^{\overrightarrow{\mathbf{b}}}} \leq C 2^{\ell(g+|\mu+\nu|)} c_{2}^{m_{\ell}},
$$

where $C<1$ and $0<c_{2}<1$ are independent of the levels $\ell$ and $L$.

Next, we will derive an estimate of $\partial_{x}^{\nu} \partial_{y}^{\mu}\left(G_{\mathrm{far}}-\tilde{G}_{\mathrm{far}}\right)$ on $\operatorname{supp} \chi^{\mathbf{b}}$ for some $\mathbf{b} \in P_{\text {far }}$. By using the splitting (6.11) we obtain

$$
G_{\text {far }}-\tilde{G}_{\text {far }}=\sum_{\mathbf{b} \in P_{\text {far }}} w^{\mathbf{b}} \sum_{\overrightarrow{\mathbf{b}} \in \overrightarrow{\mathfrak{C}}^{\mathbf{b}}} \gamma^{\overrightarrow{\mathbf{b}}} \chi^{\overrightarrow{\mathbf{b}}}\left(G-\mathcal{I}^{\overrightarrow{\mathbf{b}}} G\right) .
$$

Since $\left(w^{\mathbf{b}} \gamma^{\overrightarrow{\mathbf{b}}} \chi^{\overrightarrow{\mathbf{b}}}\right)_{\substack{\overrightarrow{\mathbf{b}} \in \overrightarrow{\mathfrak{c}}^{\mathbf{b}} \mathbf{b} \\ \mathbf{b} \in P}}$ is a partition of unity, the local estimate (6.12) carries over to (cf. Example 4.7)

$$
\left\|G_{\text {far }}-\tilde{G}_{\text {far }}\right\|_{\infty, \operatorname{supp} \chi^{\mathbf{b}}} \leq \max _{\overrightarrow{\mathbf{b}} \in \overrightarrow{\mathfrak{c}} \mathbf{b}}\left\|G-\mathcal{I}^{\overrightarrow{\mathbf{b}}} G\right\|_{\infty, \operatorname{supp} \chi} \overrightarrow{\mathbf{b}} .
$$

Next, we will estimate first derivatives of the approximation, more precisely, for $\mathbf{b} \in P_{\text {far }}$ the difference

$$
\partial\left(G_{\text {far }}^{\mathbf{b}}-\tilde{G}_{\text {far }}^{\mathbf{b}}\right)=\partial \sum_{\overrightarrow{\mathbf{b}} \in \overrightarrow{\mathfrak{c}} \mathbf{b}} \gamma^{\overrightarrow{\mathbf{b}}} \chi^{\overrightarrow{\mathbf{b}}}\left(G-\mathcal{I}^{\overrightarrow{\mathbf{b}}} G\right)
$$

where $\partial$ is a first-order derivative. Leibniz' product rule yields

$$
\partial\left(G_{\mathrm{far}}^{\mathbf{b}}-\tilde{G}_{\mathrm{far}}^{\mathbf{b}}\right)=\sum_{\overrightarrow{\mathbf{b}} \in \overrightarrow{\mathfrak{C}} \mathbf{b}} \gamma^{\overrightarrow{\mathbf{b}}}\left(\partial \chi^{\overrightarrow{\mathbf{b}}}\right)\left(G-\mathcal{I}^{\overrightarrow{\mathbf{b}}} G\right)+\sum_{\overrightarrow{\mathbf{b}} \in \overrightarrow{\mathfrak{c}} \mathbf{b}} \gamma^{\overrightarrow{\mathbf{b}}} \chi^{\overrightarrow{\mathbf{b}}} \partial\left(G-\mathcal{I}^{\overrightarrow{\mathbf{b}}} G\right) .
$$

The second sum can be treated as before and, again, the local estimates carry over to the global ones:

$$
\left\|\sum_{\overrightarrow{\mathbf{b}} \in \overrightarrow{\mathfrak{C}^{\mathbf{b}}}} \gamma^{\overrightarrow{\mathbf{b}}} \chi^{\overrightarrow{\mathbf{b}}} \partial\left(G-\mathcal{I}^{\overrightarrow{\mathbf{b}}} G\right)\right\|_{\infty, \operatorname{supp} \chi^{\mathbf{b}}} \leq \max _{\overrightarrow{\mathbf{b}} \in \overrightarrow{\vec{C}^{\mathbf{b}}}}\left\|\partial\left(G-\mathcal{I}^{\overrightarrow{\mathbf{b}}} G\right)\right\|_{\infty, \operatorname{supp} \chi^{\overrightarrow{\mathbf{b}}}} .
$$

Hence, we focus in the following to the first sum in (6.17). The difficulty here is that, in a straightforward estimate, the term $\left(\partial \chi^{\overrightarrow{\mathbf{b}}}\right)$ blows up like $2^{L}$ (cf. (4.6)) while the approximation property for a block $\mathbf{b}$ with $\ell=$ level $\mathbf{b}$ decreases as $c_{2}^{L-\ell}$, which does not compensate the $2^{L}$-term in an adequate way.

The following assumption reduces technicalities. 
Assumption 6.7. The length of subsequent intervals is strictly decreasing. There exist constants $0<\underline{q} \leq \bar{q}<1$ such that

$$
0<\underline{q} \leq\left|J_{j}^{\sigma^{\prime}}\right| /\left|J_{j}^{\sigma}\right| \leq \bar{q}<1 \quad \forall \sigma \in T \backslash T_{L} \quad \forall \sigma^{\prime} \in \operatorname{sons} \sigma \quad \forall 1 \leq j \leq 3 .
$$

Assumption 6.7 implies that in (4.33b) the first alternative always holds and that the expansion order is constant for all $\sigma \in T_{\ell}$ :

$$
m_{j}^{\sigma}=m_{\ell}:=\beta+(L-\ell) \alpha \quad \forall 1 \leq j \leq 3 .
$$

The following assumption concerns the support of the cutoff functions. Let $\Gamma_{\sigma}:=\operatorname{supp} \chi^{\sigma}, \sigma \in T$.

Assumption 6.8. There exist constants $C_{3}<\infty$ and $1<C_{4}<\infty$ so that, for all $0 \leq \ell \leq L$ and any $\sigma \in T_{\ell}$,

$$
\begin{gathered}
\operatorname{diam} \Gamma_{\sigma} \leq \operatorname{diam} Q_{\sigma} \leq C_{3} h 2^{L-\ell}, \\
C_{4}^{-1} 2^{-\ell} \leq \operatorname{diam} \Gamma_{\sigma} \leq \operatorname{diam} Q_{\sigma} \leq C_{4} 2^{-\ell} .
\end{gathered}
$$

The admissibility condition controls the maximal diameters of admissible pairs of clusters. Assumption 6.9 implies that the diameters of admissible pairs of clusters are comparable with their distance.

Assumption 6.9. There exists a constant $0<C_{7} \leq 1$ such that, for all admissible blocks $(\sigma, s) \in P_{\text {far }}$, the estimate

$$
\max \left\{\operatorname{diam}\left(Q_{\sigma}\right), \operatorname{diam}\left(Q_{s}\right)\right\} \geq C_{7} 2 \eta \operatorname{dist}\left(Q_{\sigma}, Q_{s}\right)
$$

holds.

Proposition 6.10. Let $\mathbf{b}=(\sigma, s) \in P_{\text {far }}$ with $\ell:=$ level b. Assumptions 6.8 and 6.9 imply that

$$
C^{-1} 2^{-\ell} / \eta \leq \operatorname{dist}\left(Q^{\sigma}, Q^{s}\right) \leq C 2^{-\ell} / \eta .
$$

Proof. Let $\mathbf{b}$ be as in the proposition. Then Assumptions 6.8 and 6.9 imply that

$$
\operatorname{dist}\left(Q^{\sigma}, Q^{s}\right) \leq \frac{1}{2 C_{7} \eta} \max \left\{\operatorname{diam}\left(Q_{\sigma}\right), \operatorname{diam}\left(Q_{s}\right)\right\} \leq \frac{C_{4}}{2 C_{7}} \frac{2^{-\ell}}{\eta} .
$$

For the lower bound, we use the admissibility condition (4.8) and Assumption 6.8 to obtain

$$
\operatorname{dist}\left(Q^{\sigma}, Q^{s}\right) \geq \eta^{-1} \operatorname{diam}\left(Q^{\sigma} \times Q^{s}\right) \geq \eta^{-1} \operatorname{diam} Q^{\sigma} \geq C_{4}^{-1} 2^{-\ell} / \eta .
$$

We come now to the estimate of the difference $G-\mathcal{I}^{\overrightarrow{\mathbf{b}}} G$ in the first sum of (6.17).

Proposition 6.11. Let $G \in\left\{G_{1}, G_{2}\right\}$. Let $\mathbf{b} \in P_{\text {far }}$ with $\ell:=$ level $\mathbf{b}$ and $\overrightarrow{\mathbf{b}}=$ $\left(\mathbf{b}_{j}\right)_{j=\ell}^{L} \in \overrightarrow{\mathfrak{C}^{\mathbf{b}}}$ with $\mathbf{b}_{j}=\left(\sigma_{j}, s_{j}\right)$. Then, there exist constants $\delta, C>0$ independent of $\ell, k$, and $L$ such that

$$
\left\|G-\mathcal{I}^{\mathbf{b}_{k}} G\right\|_{\infty, Q^{\sigma_{k} \times Q^{s_{k}}}} \leq C\left(\eta 2^{\ell}\right)^{g+1} 2^{-k}\left(1+\frac{\delta}{\eta 2^{\ell-k}}\right)^{1-m_{k}} \quad \forall \ell \leq k \leq L .
$$


Proof. We start with the one-dimensional case. Let $J$ be an interval and $\mathcal{I}_{J}^{k}$ the $k$-th order interpolant on $J$. Then, for any $u \in C^{1}(J)$, we have for all $x \in J$,

$$
\left|\left(u-\mathcal{I}_{J}^{k} u\right)(x)\right|=\left|\int_{x_{0}}^{x}\left(u-\mathcal{I}_{J}^{k} u\right)^{\prime}\right| \leq|J|||\left(u-\mathcal{I}_{J}^{k} u\right)^{\prime} \|_{\infty, J},
$$

where $x_{0} \in J$ is some interpolation point for $\mathcal{I}_{J}^{k}$. Let $p_{k-1} \in \mathcal{P}^{k-1}$ be any polynomial of maximal degree $k-1$ and $p_{k} \in \mathcal{P}^{k}$ an antiderivative satisfying $p_{k}^{\prime}=p_{k-1}$. Then, by using the projection property of $\mathcal{I}^{k}$, we get

$$
\begin{aligned}
\left\|\left(u-\mathcal{I}_{k} u\right)^{\prime}\right\|_{\infty, J} & =\left\|\left(u-p_{k}+p_{k}-\mathcal{I}_{k} u\right)^{\prime}\right\|_{\infty, J} \\
& \leq\left\|u^{\prime}-p_{k-1}\right\|_{\infty, J}+\left\|\left(\mathcal{I}^{k}\left(p_{k}-u\right)\right)^{\prime}\right\|_{\infty, J} .
\end{aligned}
$$

The stability estimate (6.7) leads to

$$
\left\|\left(\mathcal{I}^{k}\left(p_{k}-u\right)\right)^{\prime}\right\|_{\infty, J} \leq C_{\lambda}(k+1)^{\lambda+2}\left\|u^{\prime}-p_{k-1}\right\|_{\infty, J} .
$$

Together, we have proved

$$
\left\|u-\mathcal{I}_{J}^{k} u\right\|_{\infty, J} \leq\left\{1+C_{\lambda}(k+1)^{\lambda+2}\right\}|J| \inf _{v \in \mathcal{P}^{k-1}}\left\|u^{\prime}-v\right\|_{\infty, J} .
$$

For functions $u$, where $u^{\prime}$ satisfies the assumption of Lemma 6.2, we conclude from Lemma 6.2 that

$$
\left\|u-\mathcal{I}_{J}^{k} u\right\|_{\infty, J} \leq C C_{u^{\prime}}\left\{1+C_{\lambda}(k+1)^{\lambda+2}\right\}|J|\left(1+\frac{1}{c_{0}^{\prime}}\right)^{-k}
$$

with $c_{0}^{\prime}:=\gamma_{u^{\prime}}|J|$. In our application, $c_{0}^{\prime}$ will depend on the cluster level $\ell$ and we will assume (and prove below) uniform boundedness $c_{0}^{\prime} \leq \overline{c_{0}}$, where $\overline{c_{0}}$ is independent of $\ell$ and $L$. Under this assumption and for $k \geq 1$, we find a constant $\delta>0$ depending only on $C_{\lambda}, \overline{c_{0}}$, and $\lambda$ such that

$$
\left\|u-\mathcal{I}_{J}^{k} u\right\|_{\infty, J} \leq C C_{u^{\prime}}\left(1+\frac{1}{c_{0}^{\prime}}\right)^{1-k}|J| .
$$

By a classical tensor argument, we transfer this result to the approximation on any block $\mathbf{b}=(\sigma, s) \in P_{\text {far }}$ with $\ell:=\operatorname{level}(\mathbf{b})$. Let $\overrightarrow{\mathbf{b}}=\left(\left(\sigma_{j}, s_{j}\right)\right)_{j=\ell}^{L} \in \mathfrak{C}^{\mathbf{b}}$. Fix $\ell \leq k \leq L$. From Proposition 6.10 we derive

$$
\operatorname{diam}\left(Q^{\sigma_{k}} \times Q^{s_{k}}\right) \leq\left(C_{4} \eta 2^{k}\right)^{-1} .
$$

Let $\partial$ denote a first-order partial derivative. For the generator functions $G \in$ $\left\{G_{1}, G_{2}\right\}$ (cf. (3.1)), the estimate

$$
|\partial G|_{W^{n, \infty}\left(Q^{\sigma} \times Q^{s}\right)} \leq C_{G^{\prime}} \gamma_{G^{\prime}}^{n} n ! \forall n \in \mathbb{N} \quad \text { with } \quad C_{G^{\prime}}=C_{1}\left(\eta 2^{\ell}\right)^{g+1}, \gamma_{G^{\prime}}=C_{2} \eta 2^{\ell} .
$$

follows from (6.1) in combination with Proposition 6.10. Note that the constant $c_{0}^{\prime}$ in the multidimensional case is given (and can be estimated) by

$$
c_{0}^{\prime}:=\gamma_{G^{\prime}} \operatorname{diam}\left(Q^{\sigma_{k}} \times Q^{s_{k}}\right) \leq C_{2} / C_{4} \eta 2^{\ell-k} .
$$


Hence, we can apply (6.20) componentwise and, by a standard tensor argument, we derive

$$
\begin{aligned}
\left\|G-\mathcal{I}^{\mathbf{b}_{k}} G\right\|_{\infty, Q^{\sigma_{k} \times Q^{s_{k}}}} & \leq C\left(\eta 2^{\ell}\right)^{g+1} \operatorname{diam}\left(Q^{\sigma_{k}} \times Q^{s_{k}}\right)\left(1+\frac{\delta}{\eta 2^{\ell-k}}\right)^{1-m_{k}} \\
& \leq C\left(\eta 2^{\ell}\right)^{g+1} 2^{-k}\left(1+\frac{\delta}{\eta 2^{\ell-k}}\right)^{1-m_{k}} .
\end{aligned}
$$

Lemma 6.12. There exists $\theta>0$ independent of $\ell$ and $L$ such that, for any $\mathbf{b} \in P_{\text {far }}$ with $\ell:=$ level $\mathbf{b}$, it follows that

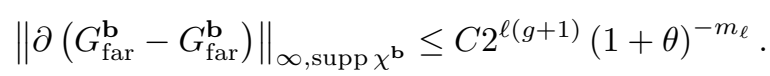

Proof. Let $\mathbf{b}=(\sigma, s) \in P_{\text {far }}$ with $\ell:=$ level b. Then,

$$
E^{\mathbf{b}}:=\sum_{\overrightarrow{\mathbf{b}} \in \overrightarrow{\mathfrak{C}} \mathbf{b}} \gamma^{\overrightarrow{\mathbf{b}}}\left(\partial \chi^{\overrightarrow{\mathbf{b}}}\right)\left(G-\mathcal{I}^{\overrightarrow{\mathbf{b}}} G\right)=\sum_{\overrightarrow{\mathbf{b}} \in \overrightarrow{\mathfrak{C}} \mathbf{b}} \gamma^{\overrightarrow{\mathbf{b}}}\left(\partial \chi^{\overrightarrow{\mathbf{b}}}\right) \sum_{k=\ell}^{L} \mathcal{I}_{k+1}^{\overrightarrow{\mathbf{b}}}\left(G-\mathcal{I}^{\mathbf{b}_{k}} G\right) .
$$

By using the inverse inequality (4.6) and Proposition 6.11 we obtain

$$
\left\|E^{\mathbf{b}}\right\|_{\infty, Q^{\sigma} \times Q^{s}} \leq C C_{\text {stab }} \sum_{\overrightarrow{\mathbf{b}} \in \overrightarrow{\mathfrak{C}^{\mathbf{b}}}} \gamma^{\overrightarrow{\mathbf{b}}} \chi_{0}^{\overrightarrow{\mathbf{b}}} 2^{L} \sum_{k=\ell}^{L} s_{k+1}^{\overrightarrow{\mathbf{b}}}\left(\eta 2^{\ell}\right)^{g+1} 2^{-k}\left(1+\frac{\delta}{\eta 2^{\ell-k}}\right)^{1-m_{k}}
$$

where, for $\overrightarrow{\mathbf{b}}=\left(\left(\sigma_{j}, s_{j}\right)\right)_{j=\ell}^{L}$ and $\ell \leq k \leq L-1$, the stability of the iterated interpolation is

$$
s_{k+1}^{\overrightarrow{\mathbf{b}}}:=\left\|\mathcal{I}_{k+1}^{\overrightarrow{\mathbf{b}}}\right\|_{L^{\infty}\left(Q^{\sigma} L \times Q^{s} L\right) \leftarrow L^{\infty}\left(Q^{\sigma} k \times Q^{s} k\right)}
$$

and $\chi_{0}^{\overrightarrow{\mathbf{b}}}: \Gamma \times \Gamma \rightarrow \mathbb{R}$ denotes the characteristic function of $\operatorname{supp} \chi^{\sigma_{L}} \times \operatorname{supp} \chi^{s_{L}}$. In [2, Theorem 3.11, Proof of Lemma 4.2] it is shown that

$$
s_{k+1}^{\mathbf{b}} \leq C \Lambda_{m_{k}}^{6}
$$

holds. Hence, by choosing $\beta \geq 1+\alpha$ in 6.19 , we obtain

$$
\begin{aligned}
\left\|E^{\mathbf{b}}\right\|_{\infty, Q^{\sigma} \times Q^{s}} & \leq C C_{\text {stab }}\left(\eta 2^{\ell}\right)^{g+1} \sum_{\overrightarrow{\mathbf{b}} \in \overrightarrow{\mathfrak{C}^{\mathbf{b}}}} \gamma^{\overrightarrow{\mathbf{b}}} \chi_{0}^{\overrightarrow{\mathbf{b}}} \sum_{k=\ell}^{L} \Lambda_{m_{k}}^{6}\left(\frac{\left(1+\frac{\delta}{\eta 2^{\ell-k}}\right)^{\alpha}}{2}\right)^{k-L-1} \\
& \leq C M C_{\text {stab }}\left(\eta 2^{\ell}\right)^{g+1} \sum_{\overrightarrow{\mathbf{b}} \in \overrightarrow{\mathfrak{C}^{\mathbf{b}}}} \gamma^{\overrightarrow{\mathbf{b}}} \chi_{0}^{\overrightarrow{\mathbf{b}}} \sum_{k=\ell}^{L} \Lambda_{m_{k}}^{6}
\end{aligned}
$$

with

$$
M:=\max _{\ell \leq k \leq L}\left(\frac{\left(1+\frac{\delta}{\eta 2^{\ell-k}}\right)^{\alpha}}{2}\right)^{k-L-1} .
$$

The maximum is attained for $k=L$, yielding

$$
M \leq \frac{2}{\left(1+\frac{\delta}{\eta 2^{\ell-L}}\right)^{\alpha}} \leq C 2^{-m_{\ell}}
$$


with $C$ depending only on $\delta, \eta$, and $\alpha$. The sum over the powers of the stability constants in (6.22) can be estimated by

$$
\sum_{k=\ell}^{L} \Lambda_{m_{k}}^{6} \leq C_{\lambda}^{6}\left(m_{\ell}+1\right)^{7} .
$$

By fixing a constant $0<\theta<1$, we obtain

$$
M \sum_{k=\ell}^{L} \Lambda_{m_{k}}^{6} \leq C(1+\theta)^{-m_{\ell}}
$$

Next, we estimate the remaining term $\sum_{\overrightarrow{\mathbf{b}} \in \overrightarrow{\mathfrak{C}} \mathbf{b}} \gamma^{\overrightarrow{\mathbf{b}}} \chi_{0}^{\overrightarrow{\mathbf{b}}}$ in $(6.22)$. Let $\mathfrak{S}^{\mathbf{b}} \in T_{L} \times T_{L}$ be the set of terminal blocks in $\overrightarrow{\mathfrak{C}}^{\mathbf{b}}$ :

$$
\mathfrak{S}^{\mathbf{b}}:=\left\{\mathbf{b}_{L}: \overrightarrow{\mathbf{b}}=\left(\mathbf{b}_{j}\right)_{j=\ell}^{L} \in \overrightarrow{\mathfrak{C}}^{\mathbf{b}}\right\}
$$

and, for $\mathbf{b}^{\prime} \in \mathfrak{S}^{\mathbf{b}}$, define $\overleftarrow{\mathfrak{C}}^{\mathbf{b}, \mathbf{b}^{\prime}} \subset \overrightarrow{\mathfrak{C}}^{\mathbf{b}}$ as

$$
\overleftarrow{\mathfrak{C}^{\mathbf{b}}, \mathbf{b}^{\prime}}:=\left\{\overrightarrow{\mathbf{b}}=\left(\mathbf{b}_{j}\right)_{j=\ell}^{L} \in \mathfrak{C}^{\mathbf{b}}: \mathbf{b}_{L}=\mathbf{b}^{\prime}\right\}
$$

Then we can write

$$
\sum_{\overrightarrow{\mathbf{b}} \in \overrightarrow{\mathfrak{C}^{\mathbf{b}}}} \gamma^{\overrightarrow{\mathbf{b}}} \chi_{0}^{\overrightarrow{\mathbf{b}}}=\sum_{\mathbf{b}^{\prime} \in \mathfrak{S}^{\mathbf{b}}} \chi_{0}^{\mathbf{b}^{\prime}} \sum_{\overrightarrow{\mathbf{b}} \in \overleftarrow{\mathfrak{C}^{\mathbf{b}}, \mathbf{b}^{\prime}}} \gamma^{\overrightarrow{\mathbf{b}}}=\sum_{\mathbf{b}^{\prime} \in \mathfrak{S}^{\mathbf{b}}} \chi_{0}^{\mathbf{b}^{\prime}} w^{\mathbf{b}^{\prime}}
$$

with $w^{\mathbf{b}} \in[0,1]$ as in (4.9). Since the supports of the basis functions have finite overlap (4.6), the right-hand side in (6.23) is bounded by a constant that is independent of the levels $\ell$ and $L$.

Altogether, we have proved

$$
\left\|E^{\mathbf{b}}\right\|_{\infty, Q^{\sigma} \times Q^{s}} \leq C(1+\theta)^{-m_{\ell}}\left(\eta 2^{\ell}\right)^{g+1} .
$$

The combination with (6.18) and Lemma 6.6 proves the assertion.

The proof in the case of second derivatives $\partial_{x} \partial_{y}\left(G_{\mathrm{far}}^{\mathbf{b}}-\tilde{G}_{\mathrm{far}}^{\mathbf{b}}\right)$ can be derived in the same fashion as for the first-order derivatives.

Remark 6.13. Since $\operatorname{supp}\left(G_{\text {far }}^{\mathbf{b}}-\tilde{G}_{\text {far }}^{\mathbf{b}}\right)=\operatorname{supp} \chi^{\mathbf{b}}$, we may replace the norm $\|\cdot\|_{\infty, \operatorname{supp} \chi^{\mathrm{b}}}$ in (6.15) and (6.21) by the norm $\|\cdot\|_{\infty, \Gamma \times \Gamma}$.

6.2. Local analysis of the kernel approximation. We have presented alternative integral equation formulations for the single and double layer potential and the hypersingular equation. For the error analysis, we will impose certain assumptions on the smoothness of the surface.

Assumption 6.14. For the

(6.24a) Single layer potential: $\Gamma$ is the surface of a Lipschitz polyhedron,

(6.24b) Double layer potential: $\Gamma$ is the surface of a Lipschitz domain and is piecewise smooth,

(6.24 $)$ Hypersingular equation: $\Gamma$ is globally smooth. 
Under these assumptions, local error estimates can be derived for the approximation of the kernels $k_{1}, k_{2}$ and $k_{3}$ (cf. (4.15), (4.16)).

The following lemma is a direct consequence of (6.12). Recall the definition of the operator $\overrightarrow{\operatorname{curl}}_{\Gamma, x y}$ as in (3.7).

Lemma 6.15. Let (6.24b) and Assumption 6.5 be satisfied. There exist $\bar{\eta}>0$ and positive constants $C_{1}<\infty$ and $c_{2}<1$ such that, for all $0<\eta<\bar{\eta}$ (cf. (4.8)), it follows that

- for all admissible blocks $\mathbf{b}=(\sigma, s)$ with $\ell=\operatorname{level}(\mathbf{b})$ and all $(x, y) \in \Gamma \times \Gamma$,

$$
\begin{gathered}
\left|k_{2}^{\mathbf{b}}(x, y)-\tilde{k}_{2}^{\mathbf{b}}(x, y)\right|+\left|\Delta_{\Gamma, x y}\left(G_{2}^{\mathbf{b}}(x, y)-\tilde{G}_{2}^{\mathbf{b}}(x, y)\right)\right| \leq \frac{C_{1} c_{2}^{m_{\ell}}}{\operatorname{dist}\left(Q_{\sigma}, Q_{s}\right)}, \\
\left|k_{1}^{\mathbf{b}}(x, y)-\tilde{k}_{1}^{\mathbf{b}}(x, y)\right| \leq \frac{C_{1} c_{2}^{m_{\ell}}}{\operatorname{dist}^{2}\left(Q_{\sigma}, Q_{s}\right)} .
\end{gathered}
$$

- For all blocks $\mathbf{b} \in P_{\text {far }}$ with $\ell=$ level $(\mathbf{b})$ and all $(x, y) \in \Gamma \times \Gamma$, we have

$$
\left|\overrightarrow{\operatorname{curl}}_{\Gamma, x y}\left(G_{2}^{\mathbf{b}}(x, y)-\tilde{G}_{2}^{\mathbf{b}}(x, y)\right)\right| \leq C_{1} c_{2}^{m_{\ell}} .
$$

- If, in addition, assumption (6.24] holds, the estimat 1

$$
\left|k_{2}^{\mathbf{b}}(x, y)-\tilde{k}_{2}^{\mathbf{b}}(x, y)\right| \leq C_{1} c_{2}^{m_{\ell}}
$$

is fulfilled for all admissible blocks $\mathbf{b}$ with $\ell=\operatorname{level}(\mathbf{b})$ and all $(x, y) \in \Gamma \times \Gamma$.

In view of this lemma, we introduce the order of singularity $g$ by

$$
g:= \begin{cases}1 & \text { if } k=k_{2} \text { or } k=k_{3}, \\ 2 & \text { if } k=k_{1}, \\ 0 & \text { if } k=k_{2} \text { and (6.24ट) holds. }\end{cases}
$$

6.3. Abstract assumptions on the mesh, the cluster tree and the block covering. The following abstract assumptions concern the covering $P$. They are proved for shape regular and quasi-uniform meshes in [25].

Definition 6.16. The uniformity of a mesh $\mathcal{G}$ is characterized by the smallest constant $C_{u}$ satisfying

where $h$ is as in (2.5) and

$$
h \leq C_{u} h_{\tau}, \quad \forall \tau \in \mathcal{G}
$$

$$
h_{\tau}:=\operatorname{diam} \tau .
$$

Definition 6.17. The shape regularity of panels is characterized by the smallest constant $C_{q}$ satisfying

$$
h_{\tau}^{2} \leq C_{q}|\tau|, \quad \forall \tau \in \mathcal{G} .
$$

Remark 6.18. Since $\mathcal{G}$ only contains finitely many panels, the constants $C_{u}, C_{q}$ are always bounded. However, it will turn out that the constants in the estimates below behave critically with increasing values of $C_{q}, C_{u}$ and we assume here that $C_{q}$ and $C_{u}$ are of moderate size.

Assumption 6.19. The constants $\alpha, \beta$ in (4.33) are chosen so that $\underline{\alpha}$ in (6.14) satisfies $\underline{\alpha}>1$ and $2^{2-g} c_{2}^{\alpha}=: C_{5}<1 / 2$ holds with $c_{2}$ as in Assumption 6.15 and $g$ as in (6.28).

\footnotetext{
${ }^{1}$ Estimate 6.27 can be improved with a substantial increase of technicalities to an upper bound $C_{1} c_{2}^{m_{\ell}} \operatorname{dist}\left(Q_{\sigma}, Q_{s}\right)$. However, it will turn out that (6.27) is sufficient for our applications.
} 


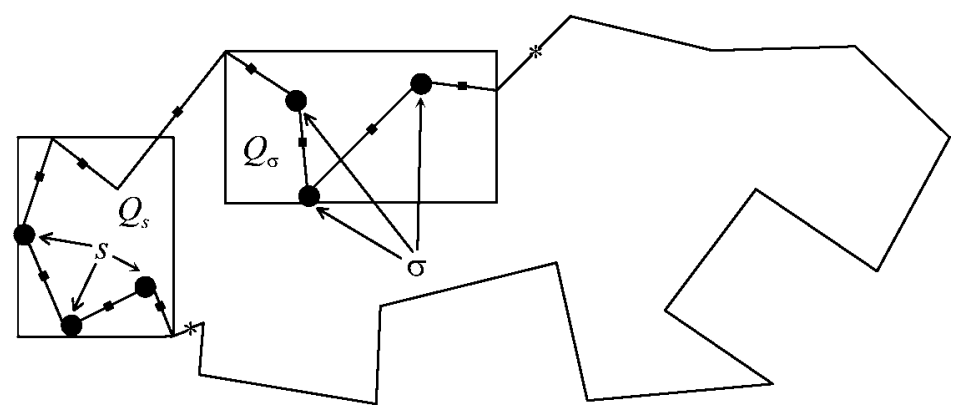

FiguRE 2. Illustration of the sets $\mathcal{G}(\mathbf{b})$ and $\mathcal{G}^{\star}(\mathbf{b})$ for a twodimensional domain with polygonal boundary. The panels are the segments of the polygon. The panels in $\mathcal{G}(\mathbf{b})$ are marked by a black square while the additional panels in $\mathcal{G}^{\star}(\mathbf{b})$ are marked by an asterisk.

We need an assumption estimating, for $\sigma \in T_{\ell}$, the number of clusters $s$ forming a block $(\sigma, s)$ in $P_{\text {far }}$.

Assumption 6.20. There exists a positive constant $C_{6}<\infty$ so that

$$
\max _{\sigma \in T} \sharp P_{\text {far }}(\sigma)=C_{6} .
$$

Note that the symmetry of the admissibility condition (cf. (4.8)) implies that

$$
\max _{\sigma \in T} \sharp\left\{\tilde{\sigma} \in T: \sigma \in P_{\text {far }}(\tilde{\sigma})\right\}=C_{6} .
$$

In order to estimate functions in the $H^{1}(\Gamma)$-norm on blocks $(\sigma, s) \in P_{\text {far }}$, we have to introduce neighbourhoods of $\sigma \cup s$ that are connected (cf. Figure 2). More precisely, we will approximate derivatives of functions on $\Gamma$ by difference quotients with respect to the surface metric and express the geodetic distance (approximately) by triangle neighbourhoods.

Notation 6.21. A sequence $\left(\tau_{i}\right)_{i=0}^{q}$ of triangles in $\mathcal{G}$ is "edge-connected" if, for all $1 \leq i \leq q$, the triangles $\tau_{i-1}$ and $\tau_{i}$ share a common edge.

For $\mathbf{b}=(\sigma, s) \in P_{\text {far }}$, let $\mathcal{G}(\mathbf{b}) \subset \mathcal{G}$ denote a smallest set having the properties

a. all triangles $\tau \in \mathcal{G}$ with $\left|\tau \cap\left(Q_{\sigma} \cup Q_{s}\right)\right|>0$ are contained in $\mathcal{G}(\mathbf{b})$,

b. for any two triangles $\tau, t \in \mathcal{G}(\mathbf{b})$, there exists an edge-connected sequence $\left(\tau_{i}\right)_{i=0}^{q}$ of triangles in $\mathcal{G}(\mathbf{b})$ with $\tau_{0}=\tau$ and $\tau_{q}=t$.

Notation 6.22. The union of all elements in $\mathcal{G}(\mathbf{b})$ defines an edge-connected neighbourhood of $\left(Q_{\sigma} \cup Q_{s}\right) \cap \Gamma$ :

$$
\mathcal{U}(\mathbf{b}):=\bigcup_{\tau \in \mathcal{G}(\mathbf{b})} \bar{\tau}
$$

Furthermore, we need the extended sets $\mathcal{G}^{\star}(\mathbf{b})$ and $\mathcal{U}^{\star}(\mathbf{b})$ defined by

$$
\mathcal{G}^{\star}(\mathbf{b}):=\{\tau \in \mathcal{G}: \bar{\tau} \cap \mathcal{U}(\mathbf{b}) \neq \emptyset\} \quad \text { and } \quad \mathcal{U}^{\star}(\mathbf{b}):=\bigcup_{\tau \in \mathcal{G}^{\star}(\mathbf{b})} \bar{\tau} .
$$

Assumption 6.23. For $\sigma \in T$, let

$$
\mathcal{U}^{\star}(\sigma):=\bigcup_{s \in P_{\text {far }}(\sigma)} \mathcal{U}^{\star}(\sigma, s)
$$


There is a positive constant $C_{8}<\infty$ such that, for all $\sigma \in T_{\ell}$, it follows that

$$
\sharp\left\{s \in T_{\ell}: \sigma \subset \mathcal{U}^{\star}(s)\right\} \leq C_{8} .
$$

Lemma 6.24. Let Assumptions 6.5, 6.8, 6.19, and 6.9 be satisfied. There exists a constant $C_{9}<\infty$ so that, for all $\mathbf{b}=(\sigma, s) \in P_{\text {far }}$ with $\ell:=$ level (b), it follows that

$$
\left(\sqrt{\left|\Gamma_{\sigma}\right|\left|\Gamma_{s}\right|}+\left|\Gamma_{s}\right|\right) c_{2}^{m(\ell)} \operatorname{dist}^{-g}\left(Q_{\sigma}, Q_{s}\right) \leq C_{9} c_{2} \eta^{g} h^{2-g} C_{5}^{L-\ell},
$$

where $g$ is as in (6.28) and $\underline{\beta}$ is as in (6.14).

Proof. Recall that $0 \leq c_{2}<1$ from Lemma 6.15 Let $\mathbf{b}=(\sigma, s) \in P_{\text {far. }}$. Without loss of generality, we assume that

$$
\operatorname{diam} \Gamma_{\sigma}=\max \left\{\operatorname{diam} \Gamma_{\sigma}, \operatorname{diam} \Gamma_{s}\right\} .
$$

Hence,

$$
\left(\sqrt{\left|\Gamma_{\sigma}\right|\left|\Gamma_{s}\right|}+\left|\Gamma_{s}\right|\right) \leq C\left(\operatorname{diam} \Gamma_{\sigma}\right)^{2}
$$

where $C$ depends only on (the curvature of) the surface $\Gamma$. Using (4.8), Assumptions 6.8, 6.5, and 6.9 we obtain

$$
\left(\sqrt{\left|\Gamma_{\sigma}\right|\left|\Gamma_{s}\right|}+\left|\Gamma_{s}\right|\right) c_{2}^{m(\ell)} \operatorname{dist}^{-g}\left(Q_{\sigma}, Q_{s}\right) \leq C \eta^{g} h^{2-g} 2^{(L-\ell)(2-g)} c_{2}^{m(\ell)}
$$

and, by employing Assumption 6.19,

$$
\begin{aligned}
& \left(\sqrt{\left|\Gamma_{\sigma}\right|\left|\Gamma_{s}\right|}+\left|\Gamma_{s}\right|\right) c_{2}^{m(\ell)} \operatorname{dist}^{-g}\left(Q_{\sigma}, Q_{s}\right) \leq C c_{2} \frac{\beta}{g} \eta^{g} h^{2-g}\left(2^{2-g} c_{\frac{\alpha}{2}}\right)^{(L-\ell)} \\
& =C c_{2}^{\beta} \eta^{g} h^{2-g} C_{5}^{L-\ell} \text {. }
\end{aligned}
$$

6.4. Quasi-interpolants. For the error analysis of the panel-clustering approximation, we will need some results concerning the approximation of functions in $H^{1}(\Gamma)$. Let $P: H^{1}(\Gamma) \rightarrow \mathcal{S}:=\mathcal{S}(0,0, \mathcal{G})$ denote the $L^{2}$-projection:

$$
\left.(P u)\right|_{\tau}=\frac{1}{|\tau|} \int_{\tau} u d s \quad \text { for all } \tau \in \mathcal{G} .
$$

Lemma 6.25. For $u \in H^{1}(\Gamma)$, let $U:=P u$ denote the $L^{2}$-projection as in (6.30). Then,

$$
\|u-U\|_{L^{2}(\Gamma)} \leq C h\|u\|_{H^{1}(\Gamma)} .
$$

For all $\mathbf{b} \in P_{\text {far }}$, it follows that

$$
\sup _{(x, y) \in \mathbf{b}}|U(y)-U(x)| \leq C|u|_{H^{1}\left(\mathcal{U}^{\star}(\mathbf{b})\right)} .
$$

Proof. Estimate (6.31) is a standard approximation result. We omit the proof and proceed directly with (6.32).

For $\mathbf{b}=(\sigma, s) \in P_{\mathrm{far}}$, let $\Gamma_{\mathbf{b}}:=\Gamma_{\sigma} \times \Gamma_{s}=\operatorname{supp} \chi^{\sigma} \times \operatorname{supp} \chi^{s}$. Choose $\tau, t \in \mathcal{G}$ with $\tau, t \in \mathcal{G}(\mathbf{b})$ such that

$$
\sup _{(x, y) \in \Gamma_{\mathbf{b}}}|U(y)-U(x)|=\left|U_{\tau}-U_{t}\right|,
$$

where $U_{K}:=\left.U\right|_{K}$ for any $K \in \mathcal{G}$. Hence,

$$
\sup _{(x, y) \in \Gamma_{\mathbf{b}}}|U(y)-U(x)|=\frac{1}{|\tau|} \int_{\tau} u d s-\frac{1}{|t|} \int_{t} u d s .
$$


Let $P_{C l}: H^{1}(\Gamma) \rightarrow \mathcal{S}(1,1, \mathcal{G})$ denote the Clément interpolation operator (cf. [4]). For $u \in H^{1}(\Gamma)$ and $\widehat{U}=P_{C l} u$, we obtain for the difference in the right-hand side of (6.33),

$$
\begin{aligned}
\frac{1}{|\tau|} \int_{\tau} u d s-\frac{1}{|t|} \int_{t} u d s & =\frac{1}{|\tau|} \int_{\tau}(u-\widehat{U}) d s-\frac{1}{|t|} \int_{t}(u-\widehat{U}) d s \\
& +\frac{1}{|\tau|} \int_{\tau} \widehat{U} d s-\frac{1}{|t|} \int_{t} \widehat{U} d s
\end{aligned}
$$

The first difference in the right-hand side of (6.34) can be estimated by

$$
\begin{aligned}
\left|\frac{1}{|\tau|} \int_{\tau}(u-\widehat{U}) d s-\frac{1}{|t|} \int_{t}(u-\widehat{U}) d s\right| & \leq C \sum_{K \in\{\tau, t\}}\|u-\widehat{U}\|_{L^{2}(K)}|K|^{-1 / 2} \\
& \leq C|u|_{H^{1}\left(\mathcal{U}^{\star}(\mathbf{b})\right)},
\end{aligned}
$$

and we proceed with the second difference in (6.34). Choose an edge-connected sequence $\left(\tau_{i}\right)_{i=0}^{q}$ with $\tau_{0}=\tau$ and $\tau_{q}=t$ and a sequence of points $M_{i} \in \overline{\tau_{i}} \cap \overline{\tau_{i-1}}$. Then,

$$
\begin{aligned}
\frac{1}{|\tau|} \int_{\tau} \widehat{U} d s & =\frac{1}{|\tau|} \int_{\tau}\left(\widehat{U}\left(M_{1}\right)+\left\langle\nabla \widehat{U}_{\tau}, y-M_{1}\right\rangle\right) d s \\
& =\widehat{U}\left(M_{1}\right)+\frac{1}{|\tau|} \int_{\tau}\left\langle\nabla \widehat{U}_{\tau}, y-M_{1}\right\rangle d s \\
& =\widehat{U}\left(M_{q}\right)+\sum_{i=1}^{q-1}\left\langle\nabla \widehat{U}_{\tau_{i}}, M_{i}-M_{i+1}\right\rangle+\frac{1}{|\tau|} \int_{\tau}\left\langle\nabla \widehat{U}_{\tau}, y-M_{1}\right\rangle d s
\end{aligned}
$$

Consequently, by using well-established properties of the Clément interpolation we get for the second difference in the right-hand side of (6.34),

$$
\left|\frac{1}{|\tau|} \int_{\tau} \widehat{U} d s-\frac{1}{|t|} \int_{t} \widehat{U} d s\right| \leq \sum_{i=0}^{q}\left\|\nabla \widehat{U}_{\tau_{i}}\right\| h_{\tau_{i}} \leq C|\widehat{U}|_{H^{1}(\mathcal{U}(\mathbf{b}))} \leq C|u|_{H^{1}\left(\mathcal{U}^{\star}(\mathbf{b})\right)} .
$$

The combination of (6.33), (6.34), (6.35), and (6.36) finishes the proof.

The next lemma concerns an inverse inequality for piecewise constant functions.

Lemma 6.26. There exists a constant $C$ depending only on the constants $C_{u}$ and $C_{q}$ (cf. Definitions 6.17 and 6.16) such that

$$
\|U\|_{L^{2}(\Gamma)} \leq C h^{-1 / 2}\|U\|_{H^{-1 / 2}(\Gamma)} \quad \forall U \in \mathcal{S}(0,0, \mathcal{G})
$$

For a proof, we refer to [5, Theorem 4.7] and [10], where the assumptions on the mesh are even relaxed.

6.5. Single layer potential operator. We consider the discretisation of the single layer potential operator by piecewise constant boundary elements $\mathcal{S}:=\mathcal{S}(0,0, \mathcal{G})$ on a quasi-uniform mesh $\mathcal{G}$. We assume that $\Gamma$ is a polyhedral Lipschitz surface. If the solution $u$ is in $H^{1}(\Gamma)$, the quasi-optimality of the Galerkin discretisation and the approximation property of $\mathcal{S}$ imply that

$$
\|u-U\|_{H^{-1 / 2}(\Gamma)} \leq C h^{3 / 2}\|u\|_{H^{1}(\Gamma)} .
$$


The effect of numerical integration in the nearfield part and the panel-clustering representation is studied in the framework of the first Strang Lemma.

Let $\tilde{a}_{S}: \mathcal{S} \times \mathcal{S} \rightarrow \mathbb{R}$ denote the bilinear form $a_{S}$ as in (4.19) where the nearfield integrals (related to $P_{\text {near }}$ ) are replaced by numerical quadrature and the farfield integrals (related to $P_{\text {far }}$ ) by the panel-clustering approximation. The corresponding solution is denoted by $\tilde{U}$. The solution $\tilde{U}$ exists provided the stability estimate

$$
\left|a_{S}(U, V)-\tilde{a}_{S}(U, V)\right| \leq C\|U\|_{H^{-1 / 2}(\Gamma)}\|V\|_{H^{-1 / 2}(\Gamma)}
$$

holds for all $U, V \in \mathcal{S}$ with a sufficiently small constant $0<C=O(1)$. The convergence is of optimal order provided the consistency estimate

$$
\inf _{U \in \mathcal{S}}\left|a_{S}(U, V)-\tilde{a}_{S}(U, V)\right| \leq C h^{3 / 2}\|u\|_{H^{1}(\Gamma)}\|V\|_{H^{-1 / 2}(\Gamma)}
$$

is satisfied for all $V \in \mathcal{S}$ and all $u \in H^{1}(\Gamma)$. We discuss the effect of the panelclustering approximation first. We assume that the bilinear form $(v, \rho u)$ in the definition of $a_{S}$ (cf. 4.19) is treated without further numerical approximations.

Let $E_{S}:=E_{P, S}+E_{Q, S}:=a_{S}(U, V)-\tilde{a}_{S}(U, V)$ with (cf. (6.11)

$$
\begin{aligned}
E_{P, S} & :=E_{P, S}^{I}+E_{P, S}^{I I}:=\sum_{\mathbf{b} \in P_{\mathrm{far}}} \int_{\Gamma} \int_{\Gamma}\left\{V(x) U(y)\left(k_{3}^{\mathbf{b}}(x, y)-\tilde{k}_{3}^{\mathbf{b}}(x, y)\right)\right. \\
& \left.+V(x)(U(y)-U(x))\left(k_{2}^{\mathbf{b}}(x, y)-\tilde{k}_{2}^{\mathbf{b}}(x, y)\right)\right\} d s_{y} d s_{x}
\end{aligned}
$$

and

$$
\begin{aligned}
E_{Q, S} & :=E_{Q, S}^{I}+E_{Q, S}^{I I} \\
& :=\sum_{\mathbf{b} \in P_{\text {near }}}\left(I_{\Gamma \times \Gamma}-Q_{\Gamma \times \Gamma}\right)\left((V \otimes U) k_{3}^{\mathbf{b}}+((V \otimes U)-(V U) \otimes 1) k_{2}^{\mathbf{b}}\right),
\end{aligned}
$$

where $I_{\Gamma \times \Gamma}$ denotes the integral over $\Gamma \times \Gamma$ and $Q_{\Gamma \times \Gamma}$ the quadrature approximation.

6.5.1. Panel-clustering approximation. We start with estimating the panel-clustering approximation error and begin with $E_{P, S}^{I I}$. If $\ell$ and $\mathbf{b}$ appear in the same context, they are linked by $\ell=\operatorname{level}(\mathbf{b})$.

We employ estimate (6.25) to derive

$$
\left|E_{P, S}^{I I}\right| \leq \sum_{\mathbf{b}=(\sigma, s) \in P_{\mathrm{far}}} C_{1} c_{2}^{m} \operatorname{dist}^{-1}\left(Q_{\sigma}, Q_{s}\right) \int_{\Gamma_{\sigma} \times \Gamma_{s}}|V(x)||U(y)-U(x)| d s_{x} d s_{y} .
$$

First, we establish the stability estimate in (6.37). The integral in (6.39) can be estimated by Hölder's inequality,

$$
\begin{aligned}
\int_{\Gamma_{\sigma} \times \Gamma_{s}}|V(x)||U(y)-U(x)| d s_{x} d s_{y} & \leq \sqrt{\left|\Gamma_{\sigma}\right|\left|\Gamma_{s}\right|}\|V\|_{L^{2}\left(\Gamma_{\sigma}\right)}\|U\|_{L^{2}\left(\Gamma_{s}\right)} \\
& +\left|\Gamma_{s}\right|\|V\|_{L^{2}\left(\Gamma_{\sigma}\right)}\|U\|_{L^{2}\left(\Gamma_{\sigma}\right)} \\
& \leq\left(\sqrt{\left|\Gamma_{\sigma}\right|\left|\Gamma_{s}\right|}+\left|\Gamma_{s}\right|\right)\|V\|_{L^{2}\left(\Gamma_{\sigma}\right)}\|U\|_{L^{2}\left(\Gamma_{\sigma} \cup \Gamma_{s}\right)} .
\end{aligned}
$$


Estimate (6.29) leads to

$$
\begin{aligned}
\left|E_{P, S}^{I I}\right| & \leq C_{1} C_{9} c \frac{\beta}{2} \eta h \sum_{\mathbf{b}=(\sigma, s) \in P_{\mathrm{far}}} C_{5}^{L-\ell}\|V\|_{L^{2}\left(\Gamma_{\sigma}\right)}\|U\|_{L^{2}\left(\Gamma_{\sigma} \cup \Gamma_{s}\right)} \\
& \leq C_{1} C_{9} c \frac{\beta}{2} \eta h \sum_{\ell=0}^{L} C_{5}^{L-\ell} \sum_{\sigma \in T_{\ell}}\|V\|_{L^{2}\left(\Gamma_{\sigma}\right)} \sum_{s \in P_{\mathrm{far}}(\sigma)}\|U\|_{L^{2}\left(\Gamma_{\sigma} \cup \Gamma_{s}\right)} \\
& \leq C_{1} C_{9} \sqrt{C_{6}} c \frac{\beta}{2} \eta h \sum_{\ell=0}^{L} C_{5}^{L-\ell} \sum_{\sigma \in T_{\ell}}\|V\|_{L^{2}\left(\Gamma_{\sigma}\right)}\|U\|_{L^{2}\left(\Gamma_{\sigma} \cup \mathcal{U}^{\star}(\sigma)\right)} \\
& \leq C_{1} C_{9} \sqrt{C_{6}} c \frac{\beta}{2} \eta h \sum_{\ell=0}^{L} C_{5}^{L-\ell}\|V\|_{L^{2}(\Gamma)} \sqrt{\sum_{\sigma \in T_{\ell}}\|U\|_{L^{2}\left(\Gamma_{\sigma} \cup \mathcal{U}^{\star}(\sigma)\right)}^{2}} \\
& \leq C_{1} C_{9} \sqrt{C_{6}\left(1+C_{8}\right)} \frac{\beta}{2} \eta h^{1 / 2}\|V\|_{H^{-1 / 2}(\Gamma)}\|U\|_{L^{2}(\Gamma)} \sum_{\ell=0}^{L} C_{5}^{L-\ell} \\
& \leq \frac{C_{1} C_{9}}{1-C_{5}} \sqrt{C_{6}\left(1+C_{8}\right)} \frac{\beta}{2} \eta\|V\|_{H^{-1 / 2}(\Gamma)}\|U\|_{H^{-1 / 2}(\Gamma)} \cdot
\end{aligned}
$$

Hence, for sufficiently small $0<\eta=O$ (1) or sufficiently large $0<\beta=O$ (1) (cf. (6.14) ), the stability requirements of the first Strang Lemma are satisfied for the term $E_{P, S}^{I I}$.

For consistency, we proceed in (6.39) with $U=P u$ (cf. (6.30) ) and employ (6.29) and Lemma 6.25 to obtain

$$
\begin{aligned}
\left|E_{P, S}^{I I}\right| & \leq C C_{9} \sum_{\mathbf{b}=(\sigma, s) \in P_{\text {far }}} \sqrt{\left|\Gamma_{s}\right|}\|V\|_{L^{2}(\sigma)}|u|_{H^{1}\left(\mathcal{U}^{\star}(\sigma)\right)} \eta h C_{5}^{L-\ell} \\
& \leq C C_{9} \eta h \sum_{\ell=0}^{L} C_{5}^{L-\ell} 2^{-\ell} \sum_{\sigma \in T_{\ell}}\|V\|_{L^{2}\left(\Gamma_{\sigma}\right)}|u|_{H^{1}\left(\mathcal{U}^{\star}(\sigma)\right)} .
\end{aligned}
$$

By using Assumptions 6.23 and 6.8 we get

$$
\begin{aligned}
\left|E_{P, S}^{I I}\right| & \leq C C_{9} \sqrt{C_{6}} \eta h^{2} \sum_{\ell=0}^{L} C_{5}^{L-\ell_{2}} 2^{L-\ell} \sum_{\sigma \in T_{\ell}}\|V\|_{L^{2}\left(\Gamma_{\sigma}\right)}|u|_{H^{1}\left(\mathcal{U}^{*}(\sigma)\right)} \\
& \leq C C_{9} \sqrt{C_{6}} \eta h^{2} \sum_{\ell=0}^{L}\left(2 C_{5}\right)^{L-\ell}\|V\|_{L^{2}(\Gamma)} \sqrt{\sum_{\sigma \in T_{\ell}}|u|_{H^{1}\left(\Gamma_{\sigma}\right)}^{2} \sum_{s: \sigma \subset \mathcal{U}^{\star}(s)} 1} \\
& \leq \frac{C C_{9} \sqrt{C_{6} C_{8}}}{1-2 C_{5}} \eta h^{3 / 2}\|V\|_{H^{-1 / 2}(\Gamma)}|u|_{H^{1}(\Gamma)},
\end{aligned}
$$

and this is the consistency estimate.

In the next step, we estimate the error $E_{P, S}^{I}$ and employ the representation

$$
E_{P, S}^{I}=\sum_{\mathbf{b}=(\sigma, s) \in P_{\mathrm{far}}} \int_{\Gamma_{\sigma} \times \Gamma_{s}} V(x) U(y)\left(\Delta_{x y}^{\Gamma} e_{\mathbf{b}}\right)(x, y) d s_{y} d s_{x}
$$

with

$$
e_{\mathbf{b}}(x, y):=\sum_{\overrightarrow{\mathbf{b}} \in \overrightarrow{\mathfrak{C}^{\mathbf{b}}}} \chi^{\overrightarrow{\mathbf{b}}}\left(G_{2}-\mathcal{I}^{\overrightarrow{\mathbf{b}}} G_{2}\right) .
$$


The stability estimate is completely analogous as for $E_{P, S}^{I I}$ due to (6.25) and hence is omitted. We proceed with the consistency.

Let $u \in H^{1}(\Gamma)$ and $U:=P u$ denote the $L^{2}$-projection of $u$ onto $\mathcal{S}(0,0, \mathcal{G})$ (cf. (6.30) $)$. Then,

$$
E_{P, S}^{I}=E_{P, S}^{I}(U, V)=E_{P, S}^{I}(U-u, V)+E_{P, S}^{I}(u, V) .
$$

We begin with the estimate of the first term. Analogous estimates as for the proof of stability lead, in combination with (6.31), to

$$
\begin{aligned}
\left|E_{P, S}^{I}(U-u, V)\right| & \leq C h^{1 / 2}\|V\|_{H^{-1 / 2}(\Gamma)}\|u-U\|_{L^{2}(\Gamma)} \\
& \leq C h^{3 / 2}\|V\|_{H^{-1 / 2}(\Gamma)}\|u\|_{H^{1}(\Gamma)} .
\end{aligned}
$$

We proceed with the estimate of the second term in (6.42). Since $u \in H^{1}(\Gamma)$, (each component of) the surface curl, $\overrightarrow{\operatorname{curl}}_{\Gamma} u$, is in $L^{2}(\Gamma)$, and we may employ partial integration in $\Gamma$ to the $\operatorname{curl}_{\Gamma, y}$ operator in (3.7). Due to the smoothness and localness of the integrands the boundary term vanishes and we obtain the representation

$$
E_{P, S}^{I}(u, V)=\sum_{\mathbf{b} \in P_{\text {far }}} \int_{\Gamma \times \Gamma} V(x)\left\langle\overrightarrow{\operatorname{curl}}_{\Gamma} u(y), \overrightarrow{\operatorname{curl}}_{\Gamma, x y} e_{\mathbf{b}}(x, y)\right\rangle d s_{y} d s_{x} .
$$

The integral can be estimated, for $\mathbf{b}=(\sigma, s) \in P_{\text {far }}$, by

$$
\begin{aligned}
\int_{\Gamma_{\sigma} \times \Gamma_{s}}|V|\left|\overrightarrow{\operatorname{curl}} \Gamma_{\Gamma} u\right| d s_{y} d s_{x} & \leq \int_{\Gamma_{\sigma}}|V| d s \int_{\Gamma_{s}}\left|\overrightarrow{\operatorname{curl}}_{\Gamma} u\right| d s \\
& \leq \sqrt{\left|\Gamma_{\sigma}\right|\left|\Gamma_{s}\right|}\|V\|_{L^{2}\left(\Gamma_{\sigma}\right)}|u|_{H^{1}\left(\Gamma_{s}\right)} .
\end{aligned}
$$

Combining these two estimates with Lemma 6.29) and arguing as in (6.40) results in

$$
\begin{aligned}
\left|E_{P, S}^{I}(u, V)\right| & \leq C_{1} C_{9} \frac{\beta}{2} h^{2} \sum_{\mathbf{b} \in P_{\mathrm{far}}} C_{5}^{L-\ell}\|V\|_{L^{2}\left(\Gamma_{\sigma}\right)}|u|_{H^{1}\left(\Gamma_{s}\right)} \\
& \leq \frac{C_{1} C_{9}}{1-C_{5}} \sqrt{C_{6}\left(1+C_{6}\right)} \frac{\beta}{2} h^{3 / 2}\|V\|_{H^{-1 / 2}(\Gamma)}|u|_{H^{1}(\Gamma)} .
\end{aligned}
$$

6.5.2. Estimate of the quadrature error. We begin with studying the nearfield quadrature error $E_{Q, S}^{I I}$. For the nearfield integrals we employ the coordinate transforms which are described in $[8$ to regularise the integrands and standard Gauß rules for their quadrature approximation with $m$ points per space dimension. Since we are dealing in the Galerkin boundary element method with four-dimensional integrals the amount of work for approximating a matrix entry is proportional to $O\left(\mathrm{~m}^{4}\right)$.

In [28], [17], [31], 29], error estimates have been developed for the quadrature approximation. It was shown that for a pair of panels $\tau \times t$ the quadrature error satisfies that

$$
\left|\left(I_{\tau \times t}-Q_{\tau \times t}^{m}\right)(W)\right| \leq C h^{3}(1+\delta)^{-2 m}\|W\|_{L^{\infty}\left(\Gamma_{\sigma} \times \Gamma_{s}\right)}
$$

holds for some $0<\delta=O(1)$ and any piecewise polynomial $W$. By assuming this quadrature error estimate and the inverse inequalities

$$
\|V\|_{L^{\infty}(\tau)} \leq C h^{-1}\|V\|_{L^{2}(\tau)}, \quad\|U\|_{L^{\infty}(\tau \times t)} \leq C h^{-1}\|U\|_{L^{2}(\tau \times t)},
$$


we derive, in a similar fashion as for the panel-clustering approximation, the stability estimate

$$
\begin{aligned}
\left|E_{Q, S}^{I I}\right| & \leq C h^{3}(1+\delta)^{-2 m} \sum_{\mathbf{b}=(\sigma, s) \in P_{\text {near }}}\|V\|_{L^{\infty}\left(\Gamma_{\sigma}\right)}\|U \otimes 1\|_{L^{\infty}\left(\Gamma_{\sigma} \times \Gamma_{s}\right)} \\
& \leq C \sqrt{C_{6}} h(1+\delta)^{-2 m} \sum_{\sigma \in T_{L}}\|V\|_{L^{2}\left(\Gamma_{\sigma}\right)}\|U\|_{L^{2}\left(\mathcal{U}^{\star}(\sigma)\right)} \\
& \leq C \sqrt{C_{6}\left(1+C_{8}\right)} h(1+\delta)^{-2 m}\|V\|_{L^{2}(\Gamma)}\|U\|_{L^{2}(\Gamma)} .
\end{aligned}
$$

In combination with the inverse inequality

$$
\|V\|_{L^{2}(\Gamma)}\|U\|_{L^{2}(\Gamma)} \leq C h^{-1}\|V\|_{H^{-1 / 2}(\Gamma)}\|U\|_{H^{-1 / 2}(\Gamma)}
$$

we obtain stability for sufficiently large $0 \leq m=O(1)$.

For the consistency estimates we put $U=P u$ (cf. (6.30) ) and employ Lemma 6.25 to argue as in (6.41), resulting in

$$
\begin{aligned}
\left|E_{Q, S}^{I I}\right| & \leq C h^{3}(1+\delta)^{-2 m} \sum_{\mathbf{b}=(\sigma, s) \in P_{\text {near }}}\left(\|V\|_{L^{\infty}\left(\Gamma_{\sigma}\right)} \sup _{(x, y) \in \Gamma_{\sigma} \times \Gamma_{s}}|U(y)-U(x)|\right) \\
& \leq C h^{2}(1+\delta)^{-2 m} \sum_{\mathbf{b}=(\sigma, s) \in P_{\text {near }}}\left(\|V\|_{L^{2}\left(\Gamma_{\sigma}\right)}|u|_{H^{1}\left(\mathcal{U}^{*}(\sigma)\right)}\right) \\
& \leq C \sqrt{C_{6}} h^{2}(1+\delta)^{-2 m} \sum_{\sigma \in T_{L}}\|V\|_{L^{2}\left(\Gamma_{\sigma}\right)}|u|_{H^{1}\left(\mathcal{U}^{*}(\sigma)\right)} \\
& \leq C \sqrt{C_{6} C_{8}} h^{2}(1+\delta)^{-2 m}\|V\|_{L^{2}(\Gamma)}|u|_{H^{1}(\Gamma)} \\
& \leq C \sqrt{C_{6} C_{8}} h^{3 / 2}(1+\delta)^{-2 m}\|V\|_{H^{-1 / 2}(\Gamma)}|u|_{H^{1}(\Gamma)},
\end{aligned}
$$

and this is consistent for any $m \geq 0$.

The quadrature error for the bilinear form $a_{s}^{I}$ can be analysed in a completely analogous way.

6.6. Hypersingular integral operator. The kernel functions arising in the hypersingular integral equation (4.18) are already analysed in the context of the single layer kernel. However, due to the smoothness assumption (6.24D) on the surface, we may employ (6.27) for the approximation quality of the kernel function $k^{(2)}$ to obtain, by simply repeating all steps in the analysis of the single layer potential operator, the required stability and consistency estimates. For all $U, V \in \mathcal{S}(1,1, \mathcal{G})$, it follows that

$$
\left|a_{H}(U, V)-\tilde{a}_{H}(U, V)\right| \leq C \rho_{m, \beta}\|U\|_{H^{1 / 2}(\Gamma)}\|V\|_{H^{1 / 2}(\Gamma)}
$$

with a function $\rho_{m, \beta}$ that depends on the local quadrature order $m$ of the nearfield integrals and the minimal approximation order $\beta$ of the panel-clustering approximation and satisfies $\rho_{m, \beta} \rightarrow 0$ as $m, \beta \rightarrow \infty$. In other words, the constant $C \rho_{m, \beta}$ is sufficiently small provided $m, \beta=O(1)$ are chosen sufficiently large.

The consistency estimate is derived as for the single layer potential. For all $V \in \mathcal{S}(1,1, \mathcal{G})$ and $u \in H^{2}(\Gamma)$, it follows that

$$
\left|a_{H}(U, V)-\tilde{a}_{H}(U, V)\right| \leq C h^{3 / 2}\|u\|_{H^{2}(\Gamma)}\|V\|_{H^{1 / 2}(\Gamma)} .
$$


Both estimates guarantee that the panel-clustering and quadrature approximation converges with optimal rate provided the undisturbed Galerkin method converges with optimal order $O\left(h^{3 / 2}\right)$.

6.7. Double layer potential operator. The panel-clustering error of the double layer potential has the representation

$$
E_{P, D}:=\sum_{\mathbf{b}=(\sigma, s) \in P_{\mathrm{far}}} \int_{\Gamma_{\sigma} \times \Gamma_{s}} V(x)(U(y)-U(x)) e_{1, \mathbf{b}}(x, y) d s_{y} d s_{x}
$$

with (cf. 6.11) )

$$
e_{1, \mathbf{b}}(x, y):=k_{1}^{\mathbf{b}}(x, y)-\tilde{k}_{1}^{\mathbf{b}}(x, y) .
$$

We employ estimate (6.25) to derive

$$
\left|E_{P, D}\right| \leq \sum_{\mathbf{b}=(\sigma, s) \in P_{\mathrm{far}}} C_{1} c_{2}^{m_{\ell}} \operatorname{dist}^{-2}\left(Q_{\sigma}, Q_{s}\right) \int_{\Gamma_{\sigma} \times \Gamma_{s}}|V(x)||U(y)-U(x)| d s_{x} d s_{y} .
$$

The stability estimate (cf. [6.37)

$$
\left|E_{P, D}\right| \leq \frac{C_{1} C_{9}}{1-C_{5}} \sqrt{C_{6}\left(1+C_{6}\right)} c \frac{\beta}{2} \eta^{2}\|V\|_{L^{2}(\Gamma)}\|U\|_{L^{2}(\Gamma)}
$$

is derived in a completely analogous fashion as for the single layer potential. Hence, for sufficiently small $0<\eta=O(1)$ or sufficiently large $0<\beta=O(1)$, the stability requirements of the first Strang Lemma are satisfied for this term.

For consistency, we proceed again as for the single layer potential operator. Setting $U=P u$ (cf. 6.30) leads to the consistency estimate

$$
\left|E_{P, D}\right| \leq \frac{C C_{9} \sqrt{C_{6} C_{9}}}{1-2 C_{5}} \eta h\|V\|_{L^{2}(\Gamma)}|u|_{H^{1}(\Gamma)} .
$$

The quadrature error analysis is again simply a repetition of the arguments in Section 6.5.2 and shows that the choice of $m=O(1)$ to be sufficiently large leads to a consistent and stable perturbation.

\section{Complexity analysis}

Definition 7.1. For each $\sigma \in T$, let

$$
\hat{\sigma}:=\left\{i \in\{1, \ldots, n\}: \operatorname{supp} b_{i} \cap \operatorname{supp} \chi^{\sigma} \neq \emptyset\right\} .
$$

Assumption 7.2. There is a constant $C_{L} \in \mathbb{N}$ satisfying

$$
1 \leq \# \hat{\sigma} \leq C_{L}
$$

for all $\sigma \in T_{L}$, i.e., each cutoff function $\chi^{\sigma}$ on the finest level $L$ interacts with at least one and not more than $C_{L}$ finite element basis functions.

If the cutoff functions and the basis functions are defined on the same grid, this assumption holds trivially. Note that Assumption 7.2 implies $\# T_{L} \sim n$.

Assumption 7.3. There is a constant $q \in \mathbb{R}_{>1}$ satisfying

$$
\# T_{\ell} \leq q^{\ell-L} \# T_{L}
$$

for all $\ell \in\{0, \ldots, L\}$. 
Assumption 7.3 is satisfied if, as in our case, the cutoff functions are constructed by using a regular mesh hierarchy. The combination of Assumptions 7.2 and 7.3 leads to $\# T_{\ell} \leq q^{\ell-L} n$ for all $\ell \in\{0, \ldots, L\}$.

Assumption 7.4. There is a constant $C_{\mathrm{sp}} \in \mathbb{R}_{\geq 0}$ satisfying

$$
\#\{s:(\sigma, s) \in P\} \leq C_{\mathrm{sp}}
$$

for all $\sigma \in T$.

In the context of hierarchical matrices, this property is known as sparsity, and in the context of multipole methods it describes that the size of the neighborhood list is bounded by $C_{\mathrm{sp}}$. In [11, it is shown that under very weak assumptions on the given boundary element mesh $\mathcal{T}$ the sparsity constant is moderately small.

Lemma 7.5. Let $\gamma \in \mathbb{R}_{>0}$ and $k \in \mathbb{N}$. There is a constant $C_{\mathrm{ep}} \in \mathbb{R}_{>0}$ such that

$$
\sum_{\ell=0}^{\infty}(\alpha+\beta \ell)^{k} \exp (-\gamma \ell) \leq C_{\mathrm{ep}}(\alpha+\beta)^{k}
$$

holds for all $\alpha, \beta \in \mathbb{N}$.

Proof. Bound the sum by the corresponding integral and apply partial integration.

Theorem 7.6 (Complexity). The matrices $\mathbf{N},\left(\mathbf{S}^{\mathbf{b}}\right)_{\mathbf{b} \in P_{\text {far }}},\left(\mathbf{L}^{\sigma}\right)_{\sigma \in T_{L}},\left(\mathbf{R}^{\sigma}\right)_{\sigma \in T_{L}}$ and $\left(\mathbf{B}^{\sigma^{\prime}, \sigma}\right)_{\sigma \in T, \sigma^{\prime} \in \operatorname{sons}(\sigma)}$ contain, in total, $\mathcal{O}(n)$ non-vanishing entries which can be computed in $\mathcal{O}(n)$ operations.

Proof. Due to Assumptions 7.2 and 7.4 the matrix $\mathbf{N}$ is sparse with not more than $C_{L} C_{\mathrm{sp}}$ non-zero entries per row. Since we have proved that the quadrature order for approximating the entries of $\mathbf{N}$ is fixed independent of $n$, these entries can be computed in $\mathcal{O}(1)$ operations and the matrix $\mathbf{N}$ can be constructed in $\mathcal{O}(n)$ operations.

Each $\mathbf{S}^{\mathbf{b}}$ with $\mathbf{b}=(\sigma, s) \in P_{\text {far }}$ has $\left(m^{\sigma} m^{s}\right)^{3}=(\beta+\alpha(L-\ell))^{6}$ entries, each of which can be computed by one evaluation of the kernel function. Therefore the number of all operations for building $\left(\mathbf{S}^{\mathbf{b}}\right)_{\mathbf{b} \in P_{\text {far }}}$ is bounded by

$$
\begin{aligned}
& \sum_{\ell=0}^{L} \sum_{\sigma \in T_{\ell}} \sum_{s,(\sigma, s) \in P_{\mathrm{far}}}(\beta+\alpha(L-\ell))^{6} \stackrel{A s .77 .4}{\leq} C_{\mathrm{sp}} \sum_{\ell=0}^{L} \sum_{\sigma \in T_{\ell}}(\beta+\alpha(L-\ell))^{6} \\
& \stackrel{\text { As. }}{\leq} C_{\mathrm{sp}}\left(\# T_{L}\right) \sum_{\ell=0}^{L} q^{\ell-L}(\beta+\alpha(L-\ell))^{6} \\
& \stackrel{\text { As. } 7.2}{\leq} C_{\mathrm{sp}} n \sum_{\ell^{\prime}=0}^{L}\left(\frac{1}{q}\right)^{\ell^{\prime}}\left(\beta+\alpha \ell^{\prime}\right)^{6} \\
& \stackrel{\text { Lm. }}{\leq .5} C_{\mathrm{sp}} C_{\mathrm{ep}} n(\beta+\alpha)^{6} .
\end{aligned}
$$

We can proceed in a similar fashion to prove that the matrices $\left(\mathbf{B}^{\sigma^{\prime}, \sigma}\right)_{\sigma \in T, \sigma^{\prime} \in \operatorname{sons}(\sigma)}$ can be computed in $\mathcal{O}(n)$ operations.

The matrices $\left(\mathbf{L}^{\sigma}\right)_{\sigma \in T_{L}}$ and $\left(\mathbf{R}^{\sigma}\right)_{\sigma \in T_{L}}$ each have $(\# \hat{\sigma})\left(m^{\sigma}\right)^{3}=(\# \hat{\sigma}) \beta^{3}$ entries. Each entry can be computed by integrating the product of a basis function (or its derivative) and a Lagrange polynomial (or its derivative) over a fixed small number 
of panels. On each panel, the integrand is a polynomial of order $\leq m^{\sigma}+2$, so it can be evaluated in $\mathcal{O}\left(\left(m^{\sigma}+2\right)^{3}\right)$ operations, leading to a bound of $\mathcal{O}\left((\# \hat{\sigma}) \beta^{3}(\beta+2)^{3}\right)=$ $\mathcal{O}(1)$ for each matrix by Assumption [7.2. We have to build $\# T_{L} \leq n$ matrices, so the total number of operations is again in $\mathcal{O}(n)$.

\section{NOTE ADDED IN PROOF}

Recently we have learned that similar formulae for regularizing boundary integral equations have been used in the following two works.

- D.R. Wilton, S.M. Rao, A.W. Glisson, D.H. Schaubert, O.M. Al-Bundak, C.M. Butler. Potential integrals for uniform and linear source distributions on polygonal and polyhedral domains. IEEE Transactions on Antennas and Propagation, vol. AP-32, no. 3, 275-281, March 1984.

- P. Ylä-Oijala, M. Taskinen. Calculation of CFIE impedance matrix elements with RWG and $n \times$ RWG functions. IEEE Transactions on Antennas and Propagation, vol. 52, no. 8, 1837-1846, August 2003.

\section{ACKNOWLEDGMENT}

Thanks are due to J.-C. Nédélec for fruitful discussions concerning the regularization of boundary integral operators.

\section{REFERENCES}

1. R. Bank and J. Xu. An Algorithm for Coarsening Unstructured Meshes. Numer. Math., 73(1):1-36, 1996. MR1379277 (97c:65055)

2. S. Börm, M. Löhndorf, and M. Melenk. Approximation of integral operators by variable-order interpolation. To appear in Numer. Math.

3. S. Börm, N. Krzebek, and S. A. Sauter. May the singular integrals in BEM be replaced by Zero? Technical Report 86, Max-Planck-Institut, Leipzig, Germany, 2003. To appear in Comp. Meth. Appl. Mech. Eng.

4. P. Clément. Approximation by Finite Element Functions using Local Regularization. RAIRO, Sér. Rouge Anal. Numér., R-2:77-84, 1975. MR0400739|(53:4569)

5. W. Dahmen, B. Faermann, I. Graham, W. Hackbusch, and S. Sauter. Inverse Inequalities on Non-Quasiuniform Meshes and Application to the Mortar Element Method. Technical Report 24, Max-Planck-Institut, Leipzig, Germany, 2001. Math Comp. 73:1107-1138, 2004. MR2047080

6. R. DeVore and G. Lorentz. Constructive Approximation. Springer-Verlag, New York, 1993. MR1261635 (95f:41001)

7. J. Elschner. The Double Layer Potential Operator over Polyhedral Domains II: Spline Galerkin Methods. Math. Meth. Appl. Sci., 15:23-37, 1992. MR1144458 (94i:65130)

8. S. Erichsen and S. Sauter. Efficient automatic quadrature in 3-d Galerkin BEM. Comp. Meth. Appl. Mech. Eng., 157:215-224, 1998. MR1634288 (99e:65163)

9. F. T. Johnson. A General Panel Method for the Analysis and Design of Arbitrary Configurations in Incompressible Flows. NASA Report 3079, NASA Ames Research Center, USA, 1980.

10. I. Graham, W. Hackbusch, and S. Sauter. Finite Elements on Degenerate Meshes: Inverse-type Inequalities and Applications. To appear in IMA J. Numer. Anal.

11. L. Grasedyck and W. Hackbusch. Construction and arithmetics of $\mathcal{H}$-matrices. Computing, 70(4):295-334, 2003. MR2011419 (2004i:65035)

12. L. Greengard and V. Rokhlin. A New Version of the Fast Multipole Method for the Laplace Equation in Three Dimensions. Acta Numerica, 6:229-269, 1997. MR.1489257 (99c:65012)

13. M. Griebel and M. Schweitzer. A Particle-Partition of Unity Method-Part III: A Multilevel Solver. SIAM J. Sci. Comp., 24(2):377-409, 2002. MR1951047 (2004e:65125)

14. W. Hackbusch. Integral Equations. ISNM. Birkhäuser, 1995. MR.1350296|(96h:45001) 
15. W. Hackbusch, C. Lage, and S. Sauter. On the Efficient Realization of Sparse Matrix Techniques for Integral Equations with Focus on Panel Clustering, Cubature and Software Design Aspects. In W. Wendland, editor, Boundary Element Topics, number 95-4, pages 51-76, Springer-Verlag, Berlin, 1997. MR1655236 (99h:65204)

16. W. Hackbusch and Z. Nowak. On the Fast Matrix Multiplication in the Boundary Element Method by Panel-Clustering. Num. Math., 54:463-491, 1989. MR0972420 (89k:65162)

17. W. Hackbusch and S. Sauter. On the Efficient Use of the Galerkin Method to Solve Fredholm Integral Equations. Applications of Mathematics, 38(4-5):301-322, 1993. MR1228511 (95c:65204)

18. W. Hackbusch and S. Sauter. Composite Finite Elements for the Approximation of PDEs on Domains with Complicated Micro-Structures. Numer. Math., 75(4):447-472, 1997. MR1431211 (97k:65251)

19. W. McLean. Strongly Elliptic Systems and Boundary Integral Equations. Cambridge, Univ. Press, 2000. MR1742312(2001a:35051)

20. J. Melenk and I.Babuška. The Partition of Unity Finite Element Method: Basic Theory andApplications. Comp. Meth. Appl. Mech. Eng., 139:289-314, 1996. MR:1426012|(97k:65258)

21. J. Nédélec. Integral Equations with Nonintegrable Kernels. Integral Equations Oper. Theory, 5:562-572, 1982. MR0665149 (84i:45011)

22. J. C. Nédélec. Acoustic and Electromagnetic Equations. Springer, New York, 2001. MR 1822275 (2002c:35003)

23. T. J. Rivlin. The Chebyshev Polynomials. Wiley, New York, 1974. MR0450850 (56:9142)

24. V. Rokhlin. Rapid solution of integral equations of classical potential theory. Journal of Computational Physics, 60(2):187-207, 1985. MR0805870 (86k:65120)

25. S. Sauter. Variable order panel clustering. Computing, 64:223-261, 2000. MR1767055 (2001e:65197)

26. S. Sauter and C. Lage. Transformation of hypersingular integrals and black-box cubature. Math. Comp., 70(97-17):223-250, 2001. MR.1803126(2001k:65053)

27. S. Sauter and C. Schwab. Randelementmethoden. Teubner, Leipzig, to appear in 2004.

28. S. A. Sauter. Über die effiziente Verwendung des Galerkinverfahrens zur Lösung Fredholmscher Integralgleichungen. Ph.D. thesis, Inst. f. Prakt. Math., Universität Kiel, 1992.

29. S. A. Sauter and C. Schwab. Quadrature for hp-Galerkin BEM in $R^{3}$. Numer. Math., 78(2):211-258, 1997. MR.1485998 (99f:65207)

30. J. Tausch and J. White. Wavelet-like Bases for Integral Equations on Surfaces with Complex Geometry. In J. Wang, M. Allen, B. Chen, and T. Mathew, editors, IMACS Series in Computational and Applied Mathematics, 1998.

31. T.v.Petersdorff and C. Schwab. Fully Discrete Multiscale Galerkin BEM. In W. Dahmen, P. Kurdila, and P. Oswald, editors, Multiscale Wavelet Methods for Partial Differential Equations, pages 287-346, New York, 1997. Academic Press. MR.1475002 (99a:65158)

MaX-Planck-Institut für Mathematik in Den Naturwissenschaften, Inselstrasse 2226, 04103 Leipzig, Germany

E-mail address: sbo@mis.mpg.de

Institut für Mathematik, Universität Zürich, Winterthurerstr. 190, CH-8057 Zürich, SWITZERLAND

E-mail address: stas@amath.unizh.ch 